\title{
ON CONVERGENCE AND CLOSEDNESS OF MULTIVALUED MARTINGALES
}

\author{
ZHEN-PENG WANG AND XING-HONG XUE
}

\begin{abstract}
In this paper, various convergence theorems and criteria of closedness of multivalued martingales, submartingales, and supermartingales are proved.
\end{abstract}

\section{INTRODUCTION AND PRELIMINARIES}

The study of multivalued functions has been developed extensively with applications in several areas of applied mathematics, such as mathematical economics, optimal control, and decision theory, (cf. Hildenbrand (1974), Himmelberg and Vleck (1974), Papageorgiou (1986), Clarke (1984), de Korvin and Kleyle (1985), Vovits, Foulk and Rose (1981), and Aubin and Frankowska (1990)). Four notions of convergence of multivalued functions in a Banach space, the Hausdorff distance convergence, the Kuratowski-Mosco convergence, the weak convergence, and the Wijsman convergence are particularly useful in the study. Illustrated by the works of de Korvin and Kleyle (1985) and Papageorgiou (1986), multivalued martingales, submartingales, and supermartingales are powerful tools in the study of convergence of random multivalued functions. In this paper we shall make a further study on convergence and closedness of multivalued martingales, submartingales, and supermartingales.

Throughout this paper $(\Omega, \mathscr{A}, P)$ is a complete probability space, $X$ is a separable Banach space with the dual $X^{*}$, and $2^{X}$ is the set of all subsets of $X$. Let

$$
\begin{gathered}
P_{c}(X)=\left\{A \in 2^{X}: A \text { is nonempty, closed, and convex }\right\}, \\
P_{c b}(X)=\left\{A \in P_{c}(X): A \text { is bounded }\right\}, \\
P_{w k c}(X)=\left\{A \in P_{c}(X): A \text { is weakly compact }\right\} .
\end{gathered}
$$

For $A \in 2^{X} \backslash \phi$, we denote by $\operatorname{cl} A$ and $\overline{\operatorname{co}} A$ the closure and the closed convex hull of $A$ respectively, and define $|A|=\sup \{\|x\|: x \in A\}$,

$$
\begin{array}{cl}
s\left(x^{*}, A\right)=\sup \left\{\left\langle x^{*}, y\right\rangle: y \in A\right\}, \quad s\left(x^{*}, \phi\right)=-\infty, & x^{*} \in X^{*}, \\
d(x, A)=\inf \{\|x-y\|: y \in A\}, \quad d(x, \phi)=\infty, & x \in X .
\end{array}
$$

Received by the editors December 19, 1991.

1991 Mathematics Subject Classification. Primary 60G42, 60G48, 60D05.

Key words and phrases. Multivalued martingale, submartingale, supermartingale, KuratowskiMosco convergence, Hausdorff distance convergence, weak convergence, Wijsman convergence, closedness of martingales, uniformly integrable, uniform sequence of subpramarts.

Research of the first author supported by the National Science Foundation of China. 
$s\left(x^{*}, A\right)$ and $d(x, A)$ are called the support function and the distance function of $A$ respectively. Let $\mathscr{F}$ be a sub- $\sigma$-algebra of $\mathscr{A}$. A random multivalued function $F$ from $\Omega \rightarrow P_{c}(X)$ is $\mathscr{F}$-measurable, if there exist $\mathscr{F}$-measurable random variables $f_{n}: \Omega \rightarrow X$,

$$
F(\omega)=\operatorname{cl}\left\{f_{n}(\omega), n \geq 1\right\}, \quad \omega \in \Omega,
$$

(cf. [4, Chapter III]). The original study of random multivalued functions goes back to Robbins $(1944,1945)$. Let $\mathscr{M}[\mathscr{F}]$ be the family of all random multivalued functions which are $\mathscr{F}$-measurable. Let

$$
\begin{gathered}
\mathscr{L}_{c}=\left\{F \in \mathscr{M}[\mathscr{A}]: F(\omega) \in P_{c}(X) \text { a.s. }\right\}, \\
\mathscr{L}_{w k c}=\left\{F \in \mathscr{L}_{c}: F(\omega) \in P_{w k c}(X) \text { a.s. }\right\}, \\
\mathscr{L}_{c}{ }^{11}=\left\{F \in \mathscr{L}_{c}: E d(0, F)<\infty\right\}, \quad \mathscr{L}_{c}^{1}=\left\{F \in \mathscr{L}_{c}: E|F|<\infty\right\} .
\end{gathered}
$$

For $F \in \mathscr{L}_{c}$, let

$$
S_{F}^{1}(\mathscr{F})=\left\{f \in L_{X}^{1}: f \text { is } \mathscr{F} \text {-measurable, } f(\omega) \in F(\omega) \text { a.s. }\right\},
$$

where $L_{X}^{1}$ is the set of all Bochner integrable r.v.'s from $\Omega \rightarrow X$. We also simplify $S_{F}^{1}(\mathscr{A})$ as $S_{F}^{1}$. It is easy to show that $S_{F}^{1}$ is nonempty if and only if $F \in \mathscr{L}_{c}^{d 1}$. The Aumann integral $E F$ of $F$ is defined by $E F=\{E f: f \epsilon$ $\left.S_{F}^{1}\right\}$, where $E f$ is the Bochner integral of $f$. The conditional expectation of $F \in \mathscr{L}_{c}^{d 1}$ with respect to $\mathscr{F}, E(F \mid \mathscr{F})$, is the unique (up to a $P$-null set) $\mathscr{F}$-measurable multivalued function in $\mathscr{L}_{c}^{d 1}$ such that

$$
S_{E(F \mid \mathscr{F})}^{1}(\mathscr{F})=\operatorname{cl}\left\{E(f \mid \mathscr{F}): f \in S_{F}^{1}\right\},
$$

the closure in $L_{X}^{1}$, (cf. [15, Theorem 5.1]). The conditional expectation of a random multivalued function behaves like the traditional single-valued conditional expectation. For example, for any $F \in \mathscr{L}_{c}^{d 1}, E(F \mid \mathscr{F})=F$ a.s., if $F$ is $\mathscr{F}$-measurable; and for any sub- $\sigma$-algebras $\mathscr{F}_{1}, \mathscr{F}_{2}$ of $\mathscr{A}$,

$$
E\left(E\left(F \mid \mathscr{F}_{1}\right) \mid \mathscr{F}_{2}\right)=E\left(F \mid \mathscr{F}_{2}\right) \text { a.s., if } \mathscr{F}_{2} \subset \mathscr{F}_{1},
$$

(cf. [15, Theorem 5.3]). For details of the definitions and properties of the measurability, the integration, and the conditional expectation of random multivalued functions, we refer the reader to the works of Castaing and Valadier (1977), Hiai and Umegaki (1977), Hiai (1985), Papageorgiou (1985a), and Aubin and Frankowska (1990). For $A, B \in 2^{X}$, let $h^{+}(A, B)=\sup \{d(a, B): a \in A\}$, $h^{-}(A, B)=\sup \{d(b, A): b \in B\}$. The Hausdorff metric $h$ on $P_{c}(X)$ is defined by

$$
h(A, B)=\max \left\{h^{+}(A, B), h^{-}(A, B)\right\}, \quad A, B \in P_{c}(X) .
$$

For $F, G \in \mathscr{L}_{c}^{1}$, define $\Delta(F, G)=E h(F, G)$. Then $\left(P_{c}(X), h\right)$ and $\left(\mathscr{L}_{c}^{1}, \Delta\right)$ are complete metric spaces (cf. Hiai and Umegaki (1977), p. 160). For $\left(A_{n}, n \geq\right.$ $1, A) \subset P_{c}(X)$, let

$$
s-\liminf A_{n}=\left\{x \in X: \lim d\left(x, A_{n}\right)=0\right\},
$$

and

$$
w-\lim \sup A_{n}=\left\{x \in X: x_{k} \stackrel{\mathrm{w}}{\rightarrow} x, \text { for some } x_{k} \in A_{n_{k}}\right\},
$$

where $\left(n_{k}, k \geq 1\right)$ is a subsequence of $(n \geq 1)$ and $\stackrel{w}{\rightarrow}$ means convergence in the weak topology of $X$. We denote by $A_{n} \stackrel{\text { K-M }}{\rightarrow} A$ the convergence of $A_{n}$ 
to $A$ in the Kuratowski-Mosco sense, if $w$ - $\lim \sup A_{n}=A=s-\lim \inf A_{n}$; by $A_{n} \stackrel{\mathrm{h}}{\rightarrow} A$ the Hausdorff distance convergence, if $h\left(A_{n}, A\right) \rightarrow 0$; and by $A_{n} \stackrel{\text { 舜 }}{\rightarrow} A$ the weak convergence, if $s\left(x^{*}, A_{n}\right) \rightarrow s\left(x^{*}, A\right)$ for each $x^{*} \in X^{*}$. We say that $A_{n}$ is Wijsman convergent to $A$ if $d\left(x, A_{n}\right) \rightarrow d(x, A), x \in X$, (cf. [10 and 19]). Since $h(A, B)=\sup \{|d(x, A)-d(x, B)|, x \in X\}$, the Hausdorff distance convergence is stronger than the Wijsman convergence. However, if (and only if) $X$ is totally bounded, these two notions of convergence coincide (cf. [8 and 33]). If restricted to $P_{c b}(X)$, then the Hausdorff distance convergence implies the Kuratowski-Mosco convergence and the weak convergence, and when $X$ is a finite dimensional space, these three types of convergence are equivalent (cf. [13, p. 165] and [30]). Clearly, for any $H \in \mathscr{L}_{c}^{1}, H(\omega) \in P_{c b}(X)$ a.s.

Let $\left(\mathscr{F}_{n}, n \geq 1\right)$ be an increasing sequence of complete sub- $\sigma$-algebras of $\mathscr{A}, \mathscr{F}_{\infty}=\sigma\left(\bigcup_{n} \mathscr{F}_{n}\right)$. By $\left(F_{n}, \mathscr{F}_{n}, n \geq 1\right)$ we mean that $\left(F_{n}\right) \subset \mathscr{L}_{c}^{d 1}$ and $F_{n}$ is $\mathscr{F}_{n}$-measurable, $n \geq 1$. Let $T$ be the set of bounded stopping times with respect to $\left(\mathscr{F}_{n}, n \geq 1\right)$ and $T(s)=\{t \in T: t \geq s\}, s \in T$.

Definition 1.1. $\left(F_{n}, \mathscr{F}_{n}, n \geq 1\right)$ is called a (multivalued) martingale, submartingale, or supermartingale, if

$$
E\left(F_{n+1} \mid \mathscr{F}_{n}\right)=, \supset \text {, or } \subset F_{n} \text { a.s., } \quad n \geq 1 .
$$

The rest of this paper is organized as follows. In $\S 1$, we shall prove various convergence results and criteria of closedness of multivalued submartingales. In $\S \S 3$ and 4 , we shall focus on convergence and closedness of multivalued martingales and supermartingales.

\section{ON MULTIVALUED SUBMARTINGALES}

Lemma 2.1. For any $F \in \mathscr{L}_{c}^{d 1}$ and sub- $\sigma$-algebra $\mathscr{F} \subset \mathscr{A}$,

(i) $|E(F \mid \mathscr{F})| \leq E(|F| \mid \mathscr{F})$ a.s.;

(ii) [12] $d(x, E(F \mid \mathscr{F})) \leq E(d(x, F) \mid \mathscr{F})$ a.s., $x \in X$;

(iii) [26] $s\left(x^{*}, E(F \mid \mathscr{F})\right)=E\left(s\left(x^{*}, F\right) \mid \mathscr{F}\right)$ a.s., $x^{*} \in X^{*}$.

Proof. For any $F \in \mathscr{L}_{c}^{d 1}$ and sub- $\sigma$-algegra $\mathscr{F} \subset \mathscr{A}$, we can choose $f_{n} \in$ $S_{E(F \mid \mathscr{F})}^{1}(\mathscr{F})$ such that $E(F \mid \mathscr{F})=\operatorname{cl}\left\{f_{n}, n \geq 1\right\}$ a.s. (cf. [15, Lemma 1.1]). It follows that for any $x \in X, x^{*} \in X^{*}$ and $\varepsilon>0$, there exists $f \in S_{F}^{1}$ such that $d(x, F) \geq\|x-f\|-\varepsilon$ a.s. and $s\left(x^{*}, F\right) \leq\left\langle x^{*}, f\right\rangle+\varepsilon$ a.s., and we can easily check that

$$
\begin{aligned}
& |E(F \mid \mathscr{F})|=\operatorname{ess~sup}_{f \in S_{E(F \mid \mathscr{F})}^{1}(\mathscr{F})}|f|=\operatorname{esssup}|E(f \mid \mathscr{F})| \\
& \leq E\left(\underset{f \in S_{F}^{1}}{\operatorname{ess} \sup ^{1}|f| \mid \mathscr{F}}\right)=E(|F| \mid \mathscr{F}) \text { a.s. } \\
& E(d(x, F) \mid \mathscr{F})=E\left(\underset{f \in S_{F}^{1}}{\operatorname{essinf}}\|x-f\| \mid \mathscr{F}\right)=\underset{f \in S_{F}^{1}}{\operatorname{essinf}} E(\|x-f\| \mid \mathscr{F}) \\
& \geq \underset{f \in S_{F}^{1}}{\operatorname{essinf}}\|x-E(f \mid \mathscr{F})\|=\underset{f \in S_{E(F \mid \mathscr{F})}^{1}(\mathscr{F})}{\operatorname{essinf}}\|x-f\| \\
& =d(x, E(F \mid \mathscr{F})) \text { a.s., } \quad x \in X,
\end{aligned}
$$


and for each $x^{*} \in X^{*}$

$$
\begin{aligned}
& s\left(x^{*}, E(F \mid \mathscr{F})\right)=\operatorname{ess~sup}_{f \in S_{E(F \mid \mathscr{F})}^{1}(\mathscr{F})}\left\langle x^{*}, f\right\rangle=\operatorname{ess} \sup _{f \in S_{F}^{1}}\left\langle x^{*}, E(f \mid \mathscr{F})\right\rangle \\
& =\underset{f \in S_{F}^{1}}{\operatorname{ess} \sup _{1}} E\left(\left\langle x^{*}, f\right\rangle \mid \mathscr{F}\right)=E\left(\underset{f \in S_{F}^{1}}{\operatorname{ess} \sup }\left\langle x^{*}, f\right\rangle \mid \mathscr{F}\right) \\
& =E\left(s\left(x^{*}, F\right) \mid \mathscr{F}\right) \quad \text { a.s. Q.E.D. }
\end{aligned}
$$

The following lemma slightly modifies Egghe's lemma [9, Lemma VIII.1.15].

Lemma 2.2. Let $I$ be a countable set and $I(n)$ a subset of $I$ such that $I(n) \subset$ $I(n+1)$ and $\bigcup_{n \geq 1} I(n)=I$. (a) $\left\{\left(x_{n}^{i}, \mathscr{F}_{n}, n \geq m\right), i \in I(m), m \geq 1\right\}$ is $a$ uniform sequence of real-valued subpramarts, i.e.,

$$
\lim _{t \in T} \sup _{s \in T(t)} \mathrm{P}\left(\sup _{i \in I(t)}\left[x_{t}^{i}-E\left(x_{s}^{i} \mid \mathscr{F}_{t}\right)\right]>\varepsilon\right)=0, \quad \varepsilon>0
$$

if and only if

$$
\lim _{t \in T} \mathrm{P}\left(\sup _{i \in I(t)}\left(x_{t}^{i}-r_{t}^{i}\right)>\varepsilon\right)=0, \quad \varepsilon>0
$$

where $I(t)=I(n)$ on $(t=n)$, and

$$
r_{n}^{i}=\underset{t \in T(n)}{\operatorname{ess} \inf _{(n)}} E\left(x_{t}^{i} \mid \mathscr{F}_{n}\right) \text { a.s., } \quad i \in I(n), n \geq 1
$$

(b) Let $\left\{\left(x_{n}^{i}, \mathscr{F}_{n}, n \geq m\right), i \in I(m), m \geq 1\right\}$ be a uniform sequence of real-valued subpramarts such that

$$
\liminf _{n} E \sup _{i \in I(n)}\left|x_{n}^{i}\right|<\infty
$$

Then for each $m \geq 1$ and $i \in I(m),\left(x_{n}^{i}, n \geq m\right)$ and $\left(r_{n}^{i}, n \geq m\right)$ converge a.s. to integrable r.v.'s $x^{i}$ and $r^{i}$ respectively, $x^{i}=r^{i}$ a.s., and

$$
\lim _{n}\left(\sup _{i \in I(n)} x_{n}^{i}\right)=\sup _{i \in I} \lim _{n} x_{n}^{i}=\sup _{i \in I} x^{i}
$$

Proof. For each $m \geq 1$ and $i \in I(m)$, it is well known that $\left(r_{n}^{i}, \mathscr{F}_{n}, n \geq m\right)$ is a generalized submartingale with values in $[-\infty, \infty)$, and it is easy to show that for any fixed $t \in T$ and $i \in I(t), r_{t}^{i}=\operatorname{essinf}_{s \in T(t)} E\left(x_{s}^{i} \mid \mathscr{F}_{t}\right)$ a.s. and there exist $\left(s_{n}^{i}, n \geq 1\right) \subset T(t)$ such that $E\left(x_{s_{n}^{i}}^{i} \mid \mathscr{F}_{t}\right) \downarrow r_{t}^{i}$ a.s., (cf. [6, Lemma 4.1 and 
Theorem 4.1]). Hence, for any $\varepsilon>0$,

$$
\begin{aligned}
\lim _{t \in T} & \sup _{s \in T(t)} \mathrm{P}\left(\sup _{i \in I(t)}\left[x_{t}^{i}-E\left(x_{s}^{i} \mid \mathscr{F}_{t}\right)\right]>\varepsilon\right) \\
\quad \leq & \lim _{t \in T} \mathrm{P}\left(\operatorname{ess} \sup _{s \in T(t)}\left(\sup _{i \in I(t)}\left[x_{t}^{i}-E\left(x_{s}^{i} \mid \mathscr{F}_{t}\right)\right]\right)>\varepsilon\right) \\
\quad & \lim _{t \in T} \mathrm{P}\left(\sup _{i \in I(t)}\left(\operatorname{ess} \sup _{s \in T(t)}\left[x_{t}^{i}-E\left(x_{s}^{i} \mid \mathscr{F}_{t}\right)\right]\right)>\varepsilon\right)=\lim _{t \in T} \mathrm{P}\left(\sup _{i \in I(t)}\left[x_{t}^{i}-r_{t}^{i}\right]>\varepsilon\right) \\
& =\lim _{t \in T} \mathrm{P}\left(\sup _{i \in I(t)}\left(\sup _{n}\left[x_{t}^{i}-E\left(x_{s_{n}^{i}}^{i} \mid \mathscr{F}_{t}\right)\right]\right)>\varepsilon\right) \\
& =\lim _{t \in T} \mathrm{P}\left(\sup _{n}\left(\sup _{i \in I(t)}\left[x_{t}^{i}-E\left(x_{s_{n}^{i}}^{i} \mid \mathscr{F}_{t}\right)\right]\right)>\varepsilon\right) \\
& =\lim _{t \in T} \sup _{n} \mathrm{P}\left(\sup _{i \in I(t)}\left[x_{t}^{i}-E\left(x_{s_{n}^{i}}^{i} \mid \mathscr{F}_{t}\right)\right]>\varepsilon\right) \\
& \leq \lim _{t \in T} \sup _{s \in T(t)} \mathrm{P}\left(\sup _{i \in I(t)}\left[x_{t}^{i}-E\left(x_{s}^{i} \mid \mathscr{F}_{t}\right)\right]>\varepsilon\right),
\end{aligned}
$$

where the last equality is based on the fact that $\sup _{i \in I(t)}\left[x_{t}^{i}-E\left(x_{s_{n}^{i}}^{i} \mid \mathscr{F}_{t}\right)\right], n \geq$ $1)$ is increasing, and (a) holds. Now assume that $\left\{\left(x_{n}^{i}, \mathscr{F}_{n}, n \geq m\right), i \in\right.$ $I(m), m \geq 1\}$ is a uniform sequence of real-valued subpramarts such that $\liminf _{n} E \sup _{i \in I(n)}\left|x_{n}^{i}\right|<\infty$. Since (2.1) is equivalent to

$$
\sup _{i \in I(n)}\left|x_{n}^{i}-r_{n}^{i}\right| \rightarrow 0 \text { a.s. }
$$

(cf. [20, Theorem 5.1]), applying Millet and Sucheston's subpramart convergence theorem [20, Theorem 5.1] and (2.3), for each $i \in I(m), m \geq 1$, $\left(x_{n}^{i}, n \geq m\right)$ and $\left(r_{n}^{i}, n \geq m\right)$ converge a.s. to integrable r.v.'s $x^{i}$ and $r^{i}$ respectively, $x^{i}=r^{i}$ a.s. To prove (2.2), first we assume that $\inf _{n \geq 1, i \in I(n)} x_{n}^{i} \geq a$ for some $a \leq 0$. Then $\inf _{n \geq 1, i \in I(n)} r_{n}^{i} \geq a$ and $\liminf _{n} E \sup _{i \in I(n)}\left|r_{n}^{i}\right| \leq$ $\liminf _{n} E \sup _{i \in I(n)}\left|x_{n}^{i}\right|+|a|<\infty$. Applying the proof of Neveu's (1972) lemma [21, Lemma 4, or 22, p. 109], $\lim _{n} \sup _{i \in I(n)} r_{n}^{i}=\sup _{i \in I} \lim _{n} r_{n}^{i}=\sup _{i \in I} r^{i}$, and by $(2.3)$,

$$
\lim _{n} \sup _{i \in I(n)} x_{n}^{i}=\lim _{n} \sup _{i \in I(n)} r_{n}^{i}=\sup _{i \in I} r^{i}=\sup _{i \in I} x^{i},
$$

(2.2) holds. In a general case, for any fixed $a \leq 0$, since

$$
\begin{aligned}
\mathrm{P}\left(\sup _{i \in I(t)}\left[x_{t}^{i} \vee a-E\left(x_{s}^{i} \vee a \mid \mathscr{F}_{t}\right)\right]>\varepsilon\right) & \leq \mathrm{P}\left(\sup _{i \in I(t)}\left[x_{t}^{i} \vee a-E\left(x_{s}^{i} \mid \mathscr{F}_{t}\right) \vee a\right]>\varepsilon\right) \\
& \leq \mathrm{P}\left(\sup _{i \in I(t)}\left[x_{t}^{i}-E\left(x_{s}^{i} \mid \mathscr{F}_{t}\right)\right]>\varepsilon\right),
\end{aligned}
$$

$\left\{\left(x_{n}^{i} \vee a, \mathscr{F}_{n}, n \geq m\right), i \in I(m), m \geq 1\right\}$ is a uniform sequence of real-valued subpramarts satisfying

$$
\liminf _{n} E \sup _{i \in I(n)}\left|x_{n}^{i} \vee a\right|<\infty
$$


Thus

$$
\begin{aligned}
\sup _{i \in I} x^{i} & \leq \lim _{n} \sup _{i \in I(n)} x_{n}^{i} \leq \lim _{n} \sup _{i \in I(n)}\left(x_{n}^{i} \vee a\right) \\
& =\sup _{i \in I} \lim _{n}\left(x_{n}^{i} \vee a\right)=\sup _{i \in I}\left(x^{i} \vee a\right) \rightarrow \sup _{i \in I} x^{i},
\end{aligned}
$$

as $a \rightarrow-\infty,(2.2)$ holds. Q.E.D.

Definition 2.1. Given $\left(F_{n}\right) \subset \mathscr{L}_{c}$, we say that (i) assumption (A) holds, if either (a) or (b) holds: (a) $X$ has the Radon-Nikodým property, (b) for some $G \in$ $\mathscr{L}_{w k c}, \liminf h_{n} h^{+}\left(F_{n}, G\right)=0$ a.s.; (ii) assumption (B) holds, if for some $G \in$ $\mathscr{L}_{w k c}, h\left(F_{n}, G \cap F_{n}\right) \rightarrow 0$ a.s.; (iii) $\left(F_{n}\right)$ is $L^{1}$-bounded, if $\sup _{n} E\left|F_{n}\right|<\infty$.

Let $\mathscr{N}$ be the set of all positive integers, $\mathscr{N}^{2}=\{(m, k): m \in \mathscr{N}, k \in \mathscr{N}\}$, and $\mathscr{N}^{2}(l)=\left\{(m, k) \in \mathscr{N}^{2}: m \leq l\right\}, l \geq 1$.

Lemma 2.3. Let $\left(F_{n}, \mathscr{F}_{n}, n \geq 1\right)$ be a submartingale. Then there exists a family of adapted sequences $\left\{\left(f_{n}^{(m, k)}, \mathscr{F}_{n}, n \geq m\right),(m, k) \in \mathscr{N}^{2}\right\} \subset L_{X}^{1}$ such that

(a) for each $n \geq 1$,

$$
\begin{gathered}
F_{n}(\omega)=\operatorname{cl}\left\{f_{n}^{(n, k)}(\omega), k \geq 1\right\}=\operatorname{cl}\left\{f_{n}^{(m, k)}(\omega), 1 \leq m \leq n, k \geq 1\right\} \quad \text { a.s. }, \\
E\left\|f_{n}^{(m, k)}-E\left(f_{n+1}^{(m, k)} \mid \mathscr{F}_{n}\right)\right\| \leq \frac{1}{2^{m+k+n+1}}, \quad n \geq m, k \geq 1,
\end{gathered}
$$

and $\left\{\left(\left\|f_{n}^{(m, k)}\right\|, \mathscr{F}_{n}, n \geq l\right),(m, k) \in \mathscr{N}^{2}(l), l \geq 1\right\}$ is a uniform sequence of subpramarts.

(b) under assumption (A), if $\sup _{n} E\left|F_{n}\right|<\infty$, then there exist r.v.'s $\left(f^{(m, k)}\right.$, $\left.(m, k) \in \mathscr{N}^{2}\right) \subset L_{X}^{1}$ such that

$$
\begin{aligned}
& f_{n}^{(m, k)} \rightarrow f^{(m, k)} \quad \text { a.s. }, \\
& \limsup _{n} d\left(x, F_{n}\right) \leq d(x, F) \text { a.s., } \quad x \in X,
\end{aligned}
$$

and (2.9) and (2.10) in Theorem 2.1 below hold, where

$$
F=\overline{\operatorname{co}}\left\{f^{(m, k)},(m, k) \in \mathscr{N}^{2}\right\} \in \mathscr{L}_{c}^{1} .
$$

Proof. Choose $\left\{f_{n}^{(n, k)}, k \geq 1\right\} \subset S_{F_{n}}^{1}\left(\mathscr{F}_{n}\right)$ such that

$$
F_{n}(\omega)=\operatorname{cl}\left\{f_{n}^{(n, k)}(\omega), k \geq 1\right\} .
$$

By the definition of multivalued submartingales and conditional expectations,

$$
S_{F_{n}}^{1}\left(\mathscr{F}_{n}\right) \subset S_{E\left(F_{n+1} \mid \mathscr{F}_{n}\right)}^{1}\left(\mathscr{F}_{n}\right)=\operatorname{cl}\left\{E\left(f \mid \mathscr{F}_{n}\right): f \in S_{F_{n+1}}^{1}\left(\mathscr{F}_{n+1}\right)\right\}
$$

Since $f_{n}^{(n, k)} \in S_{F_{n}}^{1}\left(\mathscr{F}_{n}\right)$, there is $f_{n+1}^{(n, k)} \in S_{F_{n+1}}^{1}\left(\mathscr{F}_{n+1}\right)$,

$$
E\left\|f_{n}^{(n, k)}-E\left(f_{n+1}^{(n, k)} \mid \mathscr{F}_{n}\right)\right\| \leq \frac{1}{2^{n+k+n+1}} .
$$

For each $k \geq 1$ and $m \geq 1$, by induction, we can get $f_{n}^{(m, k)} \in S_{F_{n}}^{1}\left(\mathscr{F}_{n}\right)$, $n \geq m$, such that

$$
E\left\|f_{n}^{(m, k)}-E\left(f_{n+1}^{(m, k)} \mid \mathscr{F}_{n}\right)\right\| \leq \frac{1}{2^{m+k+n+1}} .
$$


Hence, for each $(m, k) \in \mathcal{N}^{2},\left(f_{n}^{(m, k)}, \mathscr{F}_{n}, n \geq m\right)$ is a quasi-martingale i.e., $\sum_{n \geq m} E\left\|f_{n}^{(m, k)}-E\left(f_{n+1}^{(m, k)} \mid \mathscr{F}_{n}\right)\right\|<\infty$. For any $j \geq 1, t \in T(j)$ and $s \in T(t)$, let $K=\max _{\omega \in \Omega} s(\omega)$, then $(s=K) \in \mathscr{F}_{K-1}$, and for $n \geq j$ and $m \leq n$,

$$
\begin{aligned}
E\left\|f_{n}^{(m, k)}-E\left(f_{s}^{(m, k)} \mid \mathscr{F}_{n}\right)\right\| I(t=n) & \\
= & E\left\|f_{n}^{(m, k)}-E\left[f_{s \wedge(K-1)}^{(m, k)}-\left(f_{K-1}^{(m, k)}-f_{K}^{(m, k)}\right) I(s=K) \mid \mathscr{F}_{n}\right]\right\| I(t=n) \\
\leq & E\left\|f_{n}^{(m, k)}-E\left(f_{s \wedge(K-1)}^{(m, k)} \mid \mathscr{F}_{n}\right)\right\| I(t=n) \\
& \quad+E\left\|f_{K-1}^{(m, k)}-E\left(f_{K}^{(m, k)} \mid \mathscr{F}_{K-1}\right)\right\| I(t=n) \\
\leq & \cdots \leq \sum_{i \geq n} E\left\|f_{i}^{(m, k)}-E\left(f_{i+1}^{(m, k)} \mid \mathscr{F}_{i}\right)\right\| I(t=n),
\end{aligned}
$$

and

$$
\begin{aligned}
\mathbf{P}\left(\sup _{(m, k) \in \mathscr{N}^{2}(t)}\left(\left\|f_{t}^{(m, k)}\right\|-E\left(\left\|f_{s}^{(m, k)}\right\| \mid \mathscr{F}_{t}\right)\right)>\varepsilon\right) \\
\leq P\left(\sup _{(m, k) \in \mathscr{N}^{2}(t)}\left(\left\|f_{t}^{(m, k)}-E\left(f_{s}^{(m, k)} \mid \mathscr{F}_{t}\right)\right\|\right)>\varepsilon\right) \\
\leq \sum_{n \geq j} \sum_{k \geq 1} \sum_{m \leq n} P\left(\left\|f_{n}^{(m, k)}-E\left(f_{s}^{(m, k)} \mid \mathscr{F}_{n}\right)\right\| I(t=n)>\varepsilon\right) \\
\leq \sum_{n \geq j} \sum_{k \geq 1} \sum_{m \leq n} E\left\|f_{n}^{(m, k)}-E\left(f_{s}^{(m, k)} \mid \mathscr{F}_{n}\right)\right\| I(t=n) / \varepsilon \\
\leq \sum_{n \geq j} \sum_{k \geq 1} \sum_{m \leq n} \sum_{i \geq n} E\left\|f_{i}^{(m, k)}-E\left(f_{i+1}^{(m, k)} \mid \mathscr{F}_{i}\right)\right\| I(t=n) / \varepsilon \\
\leq \sum_{i \geq j} \sum_{k \geq 1} \sum_{m \leq i} \sum_{n \geq j} E\left\|f_{i}^{(m, k)}-E\left(f_{i+1}^{(m, k)} \mid \mathscr{F}_{i}\right)\right\| I(t=n) / \varepsilon \\
\leq \sum_{i \geq j} \sum_{k \geq 1} \sum_{m \leq i} E\left\|f_{i}^{(m, k)}-E\left(f_{i+1}^{(m, k)} \mid \mathscr{F}_{i}\right)\right\| / \varepsilon \\
\leq \sum_{(m, k) \in \mathscr{N}^{2}} \sum_{i=j}^{\infty} \frac{1}{2^{m+k+i+1} \varepsilon}=\frac{1}{2^{j} \boldsymbol{\varepsilon}} \rightarrow 0,
\end{aligned}
$$

as $j \rightarrow \infty$. Hence, $\left\{\left(\left\|f_{n}^{(m, k)}\right\|, \mathscr{F}_{n}, n \geq l\right),(m, k) \in \mathscr{N}^{2}(l), l \geq 1\right\}$ is a uniform sequence of subpramarts and (a) holds. If $\sup _{n} E\left|F_{n}\right|<\infty$, then

$$
\sup _{n} E \sup _{(m, k) \in \mathscr{N}^{2}(n)}\left\|f_{n}^{(m, k)}\right\|=\sup _{n} E\left|F_{n}\right|<\infty .
$$

Since $\left(f_{n}^{(m, k)}, \mathscr{F}_{n}, n \geq m\right)$ is a quasi-martingale, it is a uniform amart, i.e.,

$$
\lim _{t \in T} \sup _{s \in T(t)} E\left\|f_{t}^{(m, k)}-E\left(f_{s}^{(m, k)} \mid \mathscr{F}_{t}\right)\right\|=0
$$

(Bellow (1978)). If $X$ has the Radon-Nikodým property, (2.4) follows from Bellow's (1978) uniform amart convergence theorem. Now we assume that for some $G \in \mathscr{L}_{w k c} \liminf h_{n}\left(F_{n}, G\right)=0$ a.s. By the Riesz decomposition theorem for uniform amarts (cf. [3 and 11]), $f_{n}^{(m, k)}=h_{n}^{(m, k)}+z_{n}^{(m, k)}$, where $\left(h_{n}^{(m, k)}, \mathscr{F}_{n}, n \geq m\right)$ is a martingale and $\lim _{n} z_{n}^{(m, k)}=0$ a.s. and in $L^{1}$. 
Hence $\left(h_{n}^{(m, k)}, \mathscr{F}_{n}, n \geq m\right)$ is an $L^{1}$-bounded martingale and for a.s. $\omega \in \Omega$, $\left(h_{n}^{(m, k)}(\omega)\right)$ has a weakly accumulative point in $G(\omega)$. Applying [5, Proposition 4.4], $h_{n}^{(m, k)}$, and hence $f_{n}^{(m, k)}$, strongly converges to $f^{(m, k)} \in L_{X}^{1}$ and (2.4) holds. For each $x \in X$,

$$
\begin{aligned}
\limsup _{n} d\left(x, F_{n}\right) \\
\quad=\limsup _{n}\left\{\inf _{(m, k),\left(m^{\prime}, k^{\prime}\right) \in \mathscr{N}^{2}(n), a \in[0,1]} d\left(x, a f_{n}^{(m, k)}+(1-a) f_{n}^{\left(m^{\prime}, k^{\prime}\right)}\right)\right\} \\
\quad \leq \inf _{(m, k),\left(m^{\prime}, k^{\prime}\right) \in \mathscr{N}^{2}, a \in[0,1]} \lim _{n} d\left(x, a f_{n}^{(m, k)}+(1-a) f_{n}^{\left(m^{\prime}, k^{\prime}\right)}\right) \\
\quad=\inf _{(m, k),\left(m^{\prime}, k^{\prime}\right) \in \mathcal{N}^{2}, a \in[0,1]} d\left(x, a f^{(m, k)}+(1-a) f^{\left(m^{\prime}, k^{\prime}\right)}\right)=d(x, F) \quad \text { a.s., }
\end{aligned}
$$

(2.5) holds. By Lemma 2.2,

$$
\begin{aligned}
\lim _{n}\left|F_{n}\right| & =\lim _{n} \sup _{(m, k) \in \mathscr{N}^{2}(n)}\left\|f_{n}^{(m, k)}\right\| \\
& =\sup _{(m, k) \in \mathscr{N}^{2}} \lim _{n}\left\|f_{n}^{(m, k)}\right\|=\sup _{(m, k) \in \mathscr{N}^{2}}\left\|f^{(m, k)}\right\| \\
& =\sup _{(m, k),\left(m^{\prime}, k^{\prime}\right) \in \mathscr{N}^{2}, a \in[0,1]}\left\|a f^{(m, k)}+(1-a) f^{\left(m^{\prime}, k^{\prime}\right)}\right\|=|F| \text { a.s., }
\end{aligned}
$$

(2.9) holds, and by Fatou's lemma, $E|F| \leq \liminf _{n} E\left|F_{n}\right|<\infty, F \in \mathscr{L}_{c}^{1}$. For any fixed $x^{*} \in X^{*}$ and $(m, k) \in \mathscr{N}^{2}$, let

$$
x_{n}^{(m, k)}=\left\langle x^{*}, f_{n}^{(m, k)}\right\rangle, \quad n \geq m .
$$

Since $\left|x_{n}^{(m, k)}\right| \leq\left\|x^{*}\right\|\left\|f_{n}^{(m, k)}\right\|$ and

$$
\left|x_{t}^{(m, k)}-E\left(x_{s}^{(m, k)} \mid \mathscr{F}_{t}\right)\right| \leq\left\|x^{*}\right\|\left\|f_{t}^{(m, k)}-E\left(f_{s}^{(m, k)} \mid \mathscr{F}_{t}\right)\right\|,
$$

by (2.7) and (2.8), $\left\{\left(x_{n}^{(m, k)}, \mathscr{F}_{n}, n \geq l\right),(m, k) \in \mathscr{N}^{2}(l), l \geq 1\right\}$ is a uniform sequence of subpramarts satisfying

$$
\liminf _{n} E \sup _{(m, k) \in \mathscr{N}^{2}(n)}\left|x_{n}^{(m, k)}\right|<\infty .
$$

Applying Lemma 2.2 again,

$$
\begin{aligned}
\lim _{n} s\left(x^{*}, F_{n}\right) & =\lim _{n} \sup _{(m, k) \in \mathscr{N}^{2}(n)} x_{n}^{(m, k)} \\
& =\sup _{(m, k) \in \mathscr{N}^{2}} \lim _{n} x_{n}^{(m, k)}=\sup _{(m, k) \in \mathscr{N}^{2}}\left\langle x^{*}, f^{(m, k)}\right\rangle \\
& =\sup _{(m, k),\left(m^{\prime}, k^{\prime}\right) \in \mathscr{N}^{2}, a \in[0,1]}\left\langle x^{*}, a f^{(m, k)}+(1-a) f^{\left(m^{\prime}, k^{\prime}\right)}\right\rangle \\
& =s\left(x^{*}, F\right) \text { a.s., }
\end{aligned}
$$

(2.10) holds. Q.E.D.

We denote by $B^{*}$ the closed unit ball of $X^{*}$ and by $M^{*}$ a countable subset of $B^{*}$ which is dense in the Mackey topology. 
Lemma 2.4. Assume that $\left(F_{n}, \mathscr{F}_{n}, n \geq 1\right)$ is an $L^{1}$-bounded submartingale and assumption (A) holds. Let $F$ be the random multivalued function constructed in (2.6). If $F \in \mathscr{L}_{w k c}$, particularly, if for some $G \in \mathscr{L}_{w k c} \liminf _{n} h^{+}\left(F_{n}, G\right)=0$ a.s., then for a.s. $\omega \in \Omega, F_{n}(\omega)$ is Wijsman convergent to $F(\omega)$.

Proof. If $G \in \mathscr{L}_{w k c}$ and $\liminf _{n} h^{+}\left(F_{n}, G\right)=0$ a.s., then

$$
G \supset \overline{c o}\left\{f^{(m, k)},(m, k) \in \mathscr{N}^{2}\right\}=F \quad \text { a.s. }
$$

(since $F_{n} \ni f_{n}^{(m, k)} \rightarrow f^{(m, k)}$ a.s. as $\left.m \leq n \rightarrow \infty\right)$, and $F \in \mathscr{L}_{w k c}$. It is easy to see that for any $x \in X$ and $A \in 2^{X}$,

$$
d(x, A) \geq \sup _{x^{*} \in B^{*}}\left[\left\langle x^{*}, x\right\rangle-s\left(x^{*}, A\right)\right]
$$

and if $A \in \mathscr{L}_{w k c}$,

$$
d(x, A)=\sup _{x^{*} \in M^{*}}\left[\left\langle x^{*}, x\right\rangle-s\left(x^{*}, A\right)\right]
$$

Hence, by (2.10), which has been proved in Lemma 2.3,

$$
\begin{aligned}
\liminf _{n} d\left(x, F_{n}\right) & \geq \liminf _{n} \sup _{x^{*} \in B^{*}}\left[\left\langle x^{*}, x\right\rangle-s\left(x^{*}, F_{n}\right)\right] \\
& \geq \sup _{x^{*} \in M^{*}} \lim _{n} \inf \left[\left\langle x^{*}, x\right\rangle-s\left(x^{*}, F_{n}\right)\right] \\
& =\sup _{x^{*} \in M^{*}}\left[\left\langle x^{*}, x\right\rangle-s\left(x^{*}, F\right)\right]=d(x, F) \text { a.s. }
\end{aligned}
$$

and the a.s. Wijsman convergence follows from (2.5), the equicontinuity of $\left\{d(\cdot, A), A \in 2^{X} \backslash \phi\right\}$, and the separability of $X$. Q.E.D.

Let $\mathbf{L}_{c}^{1}$ be the closure of the set of all simple functions in $\left(\mathscr{L}_{c}^{1}, \Delta\right)$. It is easy to show that $G \in \mathbf{L}_{c}^{1}$ if and only if $G$ a.s. takes values in a separable subset of $\left(P_{c}(X), h\right)$. When $X$ is a finite dimensional space, $\mathbf{L}_{c}^{1}=\mathscr{L}_{c}^{1}$.

Theorem 2.1. Assume that $\left(F_{n}, \mathscr{F}_{n}, n \geq 1\right)$ is an $L^{1}$-bounded submartingale and assumption (A) holds. Let $F$ be the random multivalued function constructed in (2.6). Then $F \in \mathscr{L}_{c}^{1}$ and the following hold:

(a)

$$
\left|F_{n}\right| \rightarrow|F| \text { a.s. }
$$

and

$$
\lim _{n} s\left(x^{*}, F_{n}\right)=s\left(x^{*}, F\right) \quad \text { a.s., } \quad x^{*} \in X^{*} .
$$

(b) If $X^{*}$ is strongly separable or assumption (B) holds, then

$$
F_{n} \stackrel{\mathrm{w}}{\rightarrow} F \quad \text { a.s. }
$$

and

$$
F_{n} \stackrel{\mathrm{K}-\mathrm{M}}{\rightarrow} F \quad \text { a.s. }
$$

(c) If $F \in \mathscr{L}_{w k c}$, particularly, if for some $G \in \mathscr{L}_{w k c} \liminf _{n} h^{+}\left(F_{n}, G\right)=0$ a.s., then for a.s. $\omega \in \Omega, F_{n}(\omega)$ is Wijsman convergent to $F(\omega)$.

(d) If $F \in \mathbf{L}_{c}^{1}$, then

(d1) (2.12) holds; 
(d2) if (i), (ii), or (iii) holds: (i) $\left(\left|F_{n}\right|\right)$ is uniformly integrable, (ii) $X^{*}$ is strongly separable, (iii) $\left(F_{n}\right) \subset \mathbf{L}_{c}^{1}$ a.s., then for a.s. $\omega \in \Omega, F_{n}(\omega)$ is Wijsman convergent to $F(\omega)$ and $h^{+}\left(F_{n}, F\right) \rightarrow 0$ a.s.

Proof. (a) and (c) have been proved in Lemmas 2.3 and 2.4.

Proof of (b). If $X^{*}$ is strongly separable, let $X_{d}^{*}$ be a dense and countable subset of the unit ball $B^{*}$. By (2.9) and (2.10), there is a P-null set $N$ such that for each $\omega \in \Omega \backslash N$ and $x^{*} \in X_{d}^{*}$, $\sup _{n}|F(\omega)|<\infty$ and

$$
\lim _{n} s\left(x^{*}, F_{n}(\omega)\right)=s\left(x^{*}, F(\omega)\right) .
$$

Then, by a routine dense method, (2.13) holds for each $\omega \in \Omega \backslash N$ and $x^{*} \in X^{*}$, and $F_{n} \stackrel{\mathrm{W}}{\rightarrow} F$ a.s. If assumption (B) holds: $h\left(F_{n}, G \cap F_{n}\right) \rightarrow 0$ a.s. for some $G \in \mathscr{L}_{w k c}$. Then, $F \subset G$ a.s. and, by (2.10), there is a P-null set $N$ such that for each $\omega \in \Omega \backslash N$ and $x^{*} \in M^{*}$,

$$
F(\omega) \subset G(\omega), \quad \lim _{n} s\left(x^{*}, F_{n}(\omega)\right)=\lim _{n} s\left(x^{*}, F_{n}(\omega) \cap G(\omega)\right)=s\left(x^{*}, F(\omega)\right) .
$$

Since $\left(s\left(x^{*}, F_{n}(\omega) \cap G(\omega)\right), n \geq 1\right)$ is equicontinuous for the Mackey topology, hence, (2.14) holds for each $x^{*} \in X^{*}$, and (2.11) holds. Since $F_{n}$ is convex, it is easy to see that

$$
F=\overline{c o}\left\{f^{(m, k)},(m, k) \in \mathscr{N}^{2}\right\} \subset s-\liminf _{n} F_{n} \text { a.s. },
$$

and by $(2.11)$,

$$
w \text { - } \lim \sup F_{n} \subset F_{n} \subset F \quad \text { a.s. , }
$$

(cf. [14, Lemma 1.1]), (2.12) holds.

Proof of (d). We assume that $F \in \mathbf{L}_{c}^{1}$. To prove (2.12), by (2.15), we need only to show that $(2.16)$ is true. Choose $\left(X_{n}, n \geq 1\right) \subset P_{c}(X)$ such that for a.s. $\omega \in \Omega, F(\omega) \in \operatorname{cl}\left\{X_{n}, n \geq 1\right\}$, the closure in $\left(P_{c}(X), h\right)$. For each $i \geq 1$, let $\left(x_{i, j}, j \geq 1\right)$ be a dense subset in $X \backslash X_{i}$. For $i, j \geq 1$, by the separation theorem, there exists $x_{i, j}^{*} \in B^{*}$ such that

$$
\left\langle x_{i, j}^{*}, x_{i, j}\right\rangle \geq s\left(x_{i, j}^{*}, X_{i}\right)+d\left(x_{i, j}, X_{i}\right) .
$$

Choose a P-null set $N$ such that for each $\omega \in \Omega \backslash N,\left(X_{n}\right)$ is dense in the set $\{F(\omega), \omega \in \Omega \backslash N\}$, and

$$
\lim _{n} s\left(x_{i, j}^{*}, F_{n}(\omega)\right) \rightarrow s\left(x_{i, j}^{*}, F(\omega)\right), \quad i, j \geq 1 .
$$

If (2.16) is false, then there exist $\omega \in \Omega \backslash N, x \in \omega$ - $\lim \sup _{n} F_{n}(\omega) \backslash F(\omega)$, and $c>0$ such that $d(x, F(\omega))>5 c$. Choose $i \geq 1$ and $j \geq 1$ such that $h\left(X_{i}, F(\omega)\right)<c$ and $d\left(x_{i, j}, x\right)<c$. Then $d\left(x_{i, j}, X_{i}\right) \geq d\left(x_{i, j}, F(\omega)\right)-$ $h\left(X_{i}, F(\omega)\right) \geq d(x, F(\omega))-d\left(x, x_{i, j}\right)-h\left(X_{i}, F(\omega)\right)>3 c$. Since $x \in$ $w$-lim $\sup _{n} F_{n}(\omega)$, by (2.17),

$$
\begin{aligned}
& \limsup _{n} s\left(x_{i, j}^{*}, F_{n}(\omega)\right) \geq\left\langle x_{i, j}^{*}, x\right\rangle \geq\left\langle x_{i, j}^{*}, x_{i, j}\right\rangle-d\left(x_{i, j}, x\right) \\
& \quad \geq s\left(x_{i, j}^{*}, X_{i}\right)+d\left(x_{i, j}, X_{i}\right)-c \\
& \quad \geq s\left(x_{i, j}^{*}, F(\omega)\right)-h\left(X_{i}, F(\omega)\right)+2 c>s\left(x_{i, j}^{*}, F(\omega)\right)+c,
\end{aligned}
$$

which is a contradiction, and (d1) holds. Now we prove (d2). By Theorem 3.1 below, $h\left(E\left(F \mid \mathscr{F}_{n}, F\right)\right) \rightarrow 0$ a.s. 
(i) If $\left(\left|F_{n}\right|\right)$ is uniformly integrable, then, by Theorem 2.2(ii) below, $F_{n} \subset$ $E\left(F \mid \mathscr{F}_{n}\right)$ a.s., and $h^{+}\left(F_{n}, E\left(F \mid \mathscr{F}_{n}\right)\right)=0$ a.s. Hence,

$$
h^{+}\left(F_{n}, F\right) \leq h^{+}\left(F_{n}, E\left(F \mid \mathscr{F}_{n}\right)\right)+h\left(E\left(F \mid \mathscr{F}_{n}\right), F\right) \rightarrow 0 \text { a.s. }
$$

(ii) If $X^{*}$ is strongly separable, let $X_{d 1}^{*}$ be a dense and countable subset of $B^{*}$. Since for any $(A, B) \subset P_{c b}(X)$,

$$
h^{+}(A, B)=\sup _{x^{*} \in B^{*}}\left[s\left(x^{*}, A\right)-s\left(x^{*}, B\right)\right]=\sup _{x^{*} \in X_{d 1}^{*}}\left[s\left(x^{*}, A\right)-s\left(x^{*}, B\right)\right],
$$

(cf. [4, Theorem II-18]), by (2.10) and Lemmas 2.1 and 2.2, $\left(s\left(x^{*}, \mathscr{F}_{n}\right)-\right.$ $\left.s\left(x^{*}, E\left(F \mid \mathscr{F}_{n}\right)\right), \mathscr{F}_{n}, n \geq 1\right)$ is a real-valued submartingale,

$$
h^{+}\left(F_{n}, E\left(F \mid \mathscr{F}_{n}\right)\right)=\sup _{x^{*} \in X_{d_{1}^{*}}^{*}}\left[s\left(x^{*}, F_{n}\right)-s\left(x^{*}, E\left(F \mid \mathscr{F}_{n}\right)\right)\right] \rightarrow 0 \text { a.s. , }
$$

and (2.18) holds.

(iii) If $\left(F_{n}\right) \subset \mathbf{L}_{c}^{1}$ a.s., then $F_{n}$ and $E\left(F \mid \mathscr{F}_{n}\right)$ a.s. take values in a separable subset of $\left(P_{c}(X), h\right)$, since $F \in \mathbf{L}_{c}^{1}$ implies $E\left(F \mid \mathscr{F}_{n}\right) \in \mathbf{L}_{c}^{1}$. Choose $\left(X_{n}, n \geq 1\right) \subset P_{c b}(X)$ such that there is a P-null set $N,\left(X_{n}\right)$ is dense in the set $\left\{F_{n}(\omega), E\left(F \mid \mathscr{F}_{n}\right)(\omega), \omega \in \Omega \backslash N, n \geq 1\right\}$. Then we can choose a countable subset $X_{d 2}^{*}$ of $B^{*}$ such that

$$
h^{+}\left(X_{i}, X_{j}\right)=\sup _{x^{*} \in X_{d 2}^{*}}\left[s\left(x^{*}, X_{i}\right)-s\left(x^{*}, X_{j}\right)\right], \quad i, j \geq 1,
$$

and

$$
h^{+}\left(F_{n}, E\left(F \mid \mathscr{F}_{n}\right)\right)=\sup _{X_{d 2}^{*}}\left[s\left(x^{*}, F_{n}\right)-s\left(x^{*}, E\left(F \mid \mathscr{F}_{n}\right)\right)\right] \rightarrow 0 \quad \text { a.s. }
$$

(2.18) holds. Since $d\left(x, F_{n}\right) \geq d(x, F)-h^{+}\left(F_{n}, F\right)$,

$$
\liminf _{n} d\left(x, F_{n}\right) \geq d(x, F) \text { a.s., }
$$

and the a.s. Wijsman convergence follows from (2.5). Q.E.D.

Theorem 2.2. Assume that $\left(F_{n}, \mathscr{F}_{n}, n \geq 1\right)$ is an $L^{1}$-bounded submartingale and assumption (A) holds. Then the following are equivalent:

(i) $\left(\left|F_{n}\right|\right)$ is uniformly integrable;

(ii) $\left(F_{n}, \mathscr{F}_{n}, 1 \leq n \leq \infty\right)$ is a submartingale, where $F_{\infty}=F$ is constructed in (2.6);

(iii) for some $H \in \mathscr{L}_{c}^{1}, F_{n} \subset E\left(H \mid \mathscr{F}_{n}\right)$ a.s., $n \geq 1$; and if $X^{*}$ is strongly separable,

(iv) for some $H \in \mathscr{L}_{c}^{1}, s\left(x^{*}, F_{n}\right) \stackrel{L^{1}}{\rightarrow} s\left(x^{*}, H\right), x^{*} \in X^{*}$.

Proof. (i) $\Rightarrow$ (ii). We need only to show that for each $n \geq 1$,

$$
S_{F_{n}}^{1}\left(\mathscr{F}_{n}\right) \subset S_{E\left(F \mid \mathscr{F}_{n}\right)}^{1}\left(\mathscr{F}_{n}\right)
$$

where $F$ is constructed in (2.6). For any $n \geq 1, f \in S_{F_{n}}^{1}\left(\mathscr{F}_{n}\right)$ and $\varepsilon>0$, choose $l \geq n, 1 / 2^{l}<\varepsilon$. Since $F_{n} \subset E\left(F_{l} \mid \mathscr{F}_{n}\right)$, there is $g \in S_{F_{l}}^{1}\left(\mathscr{F}_{l}\right)$ such that $E\left\|E\left(g \mid \mathscr{F}_{n}\right)-f\right\|<\varepsilon$. Recall that $F_{l}=\operatorname{cl}\left\{f_{l}^{l, k}, k \geq 1\right\}$ a.s., by [14, Lemma 1.3], there exist $K \geq 1$ and an $\mathscr{F}_{l}$-measurable partition $\left\{A_{k}, 1 \leq k \leq K\right\}$ of $\Omega$ such that

$$
E\left\|g-\sum_{1 \leq k \leq K} f_{l}^{(l, k)} I\left(A_{k}\right)\right\|<\varepsilon .
$$


Let $h=\sum_{1 \leq k \leq K} f^{(l, k)} I\left(A_{k}\right)$. Then $h \in S_{F}^{1}, E\left(h \mid \mathscr{F}_{n}\right) \in S_{E\left(F \mid \mathscr{F}_{n}\right)}^{1}\left(\mathscr{F}_{n}\right)$ and

$$
\begin{aligned}
E \| f- & E\left(h \mid \mathscr{F}_{n}\right)\|\leq E\| f-E\left(g \mid \mathscr{F}_{n}\right) \| \\
& +E\left\|E\left(g \mid \mathscr{F}_{n}\right)-E\left(\sum_{1 \leq k \leq K} f_{l}^{(l, k)} I\left(A_{k}\right) \mid \mathscr{F}_{n}\right)\right\| \\
& +E\left\|E\left(\sum_{1 \leq k \leq K} f_{l}^{(l, k)} I\left(A_{k}\right) \mid \mathscr{F}_{n}\right)-E\left(h \mid \mathscr{F}_{n}\right)\right\| \\
\leq & \varepsilon+E\left\|g-\sum_{1 \leq k \leq K} f_{l}^{(l, k)} I\left(A_{k}\right)\right\| \\
& +E\left\|\sum_{1 \leq k \leq K}\left(E\left(f_{l}^{(l, k)} I\left(A_{k}\right) \mid \mathscr{F}_{n}\right)-E\left(f^{(l, k)} I\left(A_{k}\right) \mid \mathscr{F}_{n}\right)\right)\right\| \\
\leq & 2 \varepsilon+\sum_{1 \leq k \leq K} E\left\|f_{l}^{(l, k)}-E\left(f^{(l, k)} \mid \mathscr{F}_{l}\right)\right\| \\
\leq & 2 \varepsilon+\sum_{1 \leq k \leq K} \sum_{i=l}^{l+L} E\left\|f_{i}^{(l, k)}-E\left(f_{i+1}^{(l, k)} \mid \mathscr{F}_{i}\right)\right\| \\
& +\sum_{1 \leq k \leq K} E\left\|f_{l+L+1}^{(l, k)}-E\left(f^{(l, k)} \mid \mathscr{F}_{l+L+1}\right)\right\| \\
\leq & 2 \varepsilon+\sum_{1 \leq k \leq K} \sum_{i=l}^{\infty} \frac{1}{2^{i+l+k+1}}+\sum_{1 \leq k \leq K} E\left\|f_{l+L+1}^{(l, k)}-E\left(f^{(l, k)} \mid \mathscr{F}_{l+L+1}\right)\right\| \\
\leq & 2 \varepsilon+\frac{1}{2^{2 l}}+\sum_{1 \leq k \leq K} E\left\|f_{l+L+1}^{(l, k)}-E\left(f^{(l, k)} \mid \mathscr{F}_{l+L+1}\right)\right\| \\
\leq & 3 \varepsilon+\sum_{1 \leq k \leq K} E\left\|f_{l+L+1}^{(l, k)}-E\left(f^{(l, k)} \mid \mathscr{F}_{l+L+1}\right)\right\| \rightarrow 3 \varepsilon,
\end{aligned}
$$

as $L \rightarrow \infty$, since $\left(\left|F_{n}\right|\right)$ is uniformly integrable and $f_{i}^{(l, k)}$ and $E\left(f^{(l, k)} \mid f_{i}\right)$ $L^{1}$-converge to $f^{(l, k)}$. Therefore, $f \in S_{E\left(F \mid \mathscr{F}_{n}\right)}^{1}\left(\mathscr{F}_{n}\right),(2.19)$ holds. (ii) $\Rightarrow$ (iii) is clear. (iii) $\Rightarrow$ (i): For each $n \geq 1$, by Lemma 2.1, $\left|F_{n}\right| \leq\left|E\left(H \mid \mathscr{F}_{n}\right)\right| \leq$ $E\left(|H| \mid \mathscr{F}_{n}\right)$ a.s., (i) holds. By (2.10), (i) implies (iv). (iv) $\Rightarrow$ (iii): By (iv) and Lemma 2.1, $\left(s\left(x^{*}, F_{n}\right), \mathscr{F}_{n}, n \geq 1\right)$ is a closed real-valued submartingale,

$$
s\left(x^{*}, F_{n}\right) \leq E\left(s\left(x^{*}, H\right) \mid \mathscr{F}_{n}\right)=s\left(x^{*}, E\left(H \mid \mathscr{F}_{n}\right)\right) \quad \text { a.s. }, \quad x^{*} \in X^{*},
$$

which implies (iii) if $X^{*}$ is strongly separable. Q.E.D.

Remark 2.1. For an $L^{1}$-bounded martingale $\left(F_{n}, \mathscr{F}_{n}, n \geq 1\right)$, Papageorgiou (1989) proved that $\left|F_{n}\right|$ converges a.s. In Theorem 2.1, we identify the limit under assumption $(\mathrm{A})$.

Remark 2.2. Under additional conditions: $X$ is reflexive and $\left\{s\left(x^{*}, F_{n}\right), n \geq\right.$ $1\}$ is a.s. equi-lower-semicontinuous, Papageorgiou (1985b) proved that if sub- 
martingale $\left(F_{n}, \mathscr{F}_{n}, n \geq 1\right)$ is $L^{1}$-bounded, then $F_{n} \stackrel{\text { K-M }}{\rightarrow} H$ a.s. for some $H \in \mathscr{L}_{c}^{1}$; and if $\left(\left|F_{n}\right|\right)$ is uniformly integrable, then the submartingale is closed.

\section{ON MULTIVALUED MARTINGALES}

Under different assumptions, Van Cutsem (1969), Neveu (1972), Daures (1973), Hiai and Umegaki (1977), and Papageorgiou (1989) proved convergence and closedness theorems for multivalued martingales. In this section we continue this study. We begin with a multivalued martingale convergence theorem in Lévy's type.

Theorem 3.1. Suppose that $F \in \mathbf{L}_{c}^{1}$. Let $F_{n}=E\left(F \mid \mathscr{F}_{n}\right), n \geq 1$. Then $F_{n} \stackrel{\mathrm{h}}{\rightarrow} F_{\infty}$ a.s. and $\Delta\left(F_{n}, F_{\infty}\right) \rightarrow 0$, where $F_{\infty}=E\left(F \mid \mathscr{F}_{\infty}\right)$.

Proof. Without loss of generality, we may assume that $F$ is $\mathscr{F}_{\infty}$-measurable. For any $\varepsilon>0$, pick a simple function $H \in \mathscr{L}_{c}^{1}$ such that $H$ is $\mathscr{F}_{\infty}$ measurable and $\Delta(F, H)<\varepsilon^{2}$. Assume that $H=\sum_{k=1}^{K} H_{k} I\left(A_{k}\right)$, where $\bigcup_{k=1}^{K} A_{k}=\Omega$, $A_{k} A_{j}=\varnothing, k \neq j$, and $H_{k} \in P_{c b}(X)$. Pick $\delta>0$ such that

$$
\delta<\varepsilon^{2} /\left(2 K \max _{1 \leq i \leq K}\left|H_{i}\right|\right) .
$$

Choose $n_{1}<n_{2}<\cdots<n_{K}$ and $B_{k} \in \mathscr{F}_{n_{k}}$ such that $\mathrm{P}\left(B_{k} \backslash A_{k}\right)+\mathrm{P}\left(A_{k} \backslash B_{k}\right)<$ $\delta / 3 K$. Let

$$
C_{k}=B_{k} \backslash\left(\bigcup_{1 \leq j<k} B_{j}\right), \quad 1 \leq k<K, \quad C_{K}=\Omega \backslash\left(\bigcup_{1 \leq k<K} C_{k}\right),
$$

and $G=\sum_{k=1}^{K} H_{k} I\left(C_{k}\right)$. Then $\mathrm{P}\left(C_{k} \backslash A_{k}\right)+\mathrm{P}\left(A_{k} \backslash C_{k}\right)<\delta$, and

$$
\Delta(G, F) \leq \Delta(G, H)+\Delta(H, F) \leq 2 K \delta \max _{1 \leq i \leq K}\left|H_{i}\right|+\varepsilon^{2}<2 \varepsilon^{2} .
$$

For any $n \geq n_{K}$, by [14, Lemma 2.6],

$$
h\left(F_{n}, G\right)=h\left(E\left(F \mid \mathscr{F}_{n}\right), E\left(G \mid \mathscr{F}_{n}\right)\right) \leq E\left(h(F, G) \mid \mathscr{F}_{n}\right) \equiv h_{n} .
$$

Let $t=\inf \left\{n \geq n_{K}, h_{n}>\varepsilon\right\} \quad(\inf \{\phi\}=\infty)$. Then

$$
\mathrm{P}\left(\sup _{n \geq n_{K}} h_{n}>\varepsilon\right) \leq E h_{t} I(t<\infty) / \varepsilon \leq \Delta(F, G) / \varepsilon<2 \varepsilon,
$$

and

$$
\begin{aligned}
\mathrm{P}\left(\sup _{n \geq n_{K}} h\left(F_{n}, F\right)>2 \varepsilon\right) & \leq \mathrm{P}\left(\sup _{n \geq n_{K}} h\left(F_{n}, G\right)>\varepsilon\right)+\mathrm{P}(h(G, F)>\varepsilon) \\
& \leq \mathrm{P}\left(\sup _{n \geq n_{K}} h_{n}>\varepsilon\right)+2 \varepsilon<4 \varepsilon .
\end{aligned}
$$

Hence $F_{n} \stackrel{\mathrm{h}}{\rightarrow} F$ a.s. Since $h\left(F_{n}, F\right) \leq\left|F_{n}\right|+|F| \leq E\left(|F| \mid \mathscr{F}_{n}\right)+|F|$,

$$
\Delta\left(F_{n}, F\right)=E h\left(F_{n}, F\right) \rightarrow 0 \text {. Q.E.D. }
$$

Theorem 3.2. Assume that $\left(F_{n}, \mathscr{F}_{n}, n \geq 1\right)$ is an $L^{1}$-bounded martingale and assumption (A) holds. Then the following are equivalent:

(i) $\left(\left|F_{n}\right|\right)$ is uniformly integrable;

(ii) $\left(F_{n}, \mathscr{F}_{n}, 1 \leq n \leq \infty\right)$ is a martingale, where $F_{\infty}=F$ is constructed in 
(iii) for some $H \in \mathscr{L}_{c}^{1}, F_{n}=E\left(H \mid \mathscr{F}_{n}\right)$ a.s., $n \geq 1$; and if $X^{*}$ is strongly separable,

(vi) for some $H \in \mathscr{L}_{c}^{1}, s\left(x^{*}, F_{n}\right) \stackrel{L^{1}}{\rightarrow} s\left(x^{*}, H\right), x^{*} \in X^{*}$.

Proof. By Theorem 2.2 and its proof, we need only to show that (i) implies $E\left(F \mid \mathscr{F}_{n}\right) \subset F_{n}$ a.s. For any $n \geq 1, \mathscr{F}_{\infty}$-measurable partition $\left(A_{k}, 1 \leq k \leq K\right)$ of $\Omega$, and $\bar{k} \in \mathscr{N}^{2}, 1 \leq k \leq K$, there is $L \geq 1$ such that $\bar{k} \in \mathscr{N}^{2}(L), 1 \leq$ $k \leq K$. Let $f=\sum_{1 \leq k \leq K} f^{\bar{k}} I\left(A_{k}\right)$ and $f_{l}=\sum_{1 \leq k \leq K} \mathrm{P}\left(A_{k} \mid \mathscr{F}_{n+l}\right) f_{n+l}^{\bar{k}}, l \geq L$, where $f^{\bar{k}}$ and $f_{n+k}^{\bar{k}}$ are defined in Lemma 2.3. Then $E\left(f \mid \mathscr{F}_{n}\right) \in S_{E\left(F \mid \mathscr{F}_{n}\right)}^{1}\left(\mathscr{F}_{n}\right)$ and $E\left(f_{l} \mid \mathscr{F}_{n}\right) \in S_{E\left(F_{n+1} \mid \mathscr{F}_{n}\right)}^{1}\left(\mathscr{F}_{n}\right)=S_{F_{n}}^{1}\left(\mathscr{F}_{n}\right)$, since $F$ and $F_{n+l}$ are convex. By (i),

$$
\begin{aligned}
& E\left\|E\left(f \mid \mathscr{F}_{n}\right)-E\left(f_{l} \mid \mathscr{F}_{n}\right)\right\| \\
& \quad \leq E\left\|f-f_{l}\right\| \leq \sum_{1 \leq k \leq K} E\left\|f^{\bar{k}} I\left(A_{k}\right)-f_{n+l}^{\bar{k}} \mathrm{P}\left(A_{k} \mid \mathscr{F}_{n+l}\right)\right\| \rightarrow 0
\end{aligned}
$$

as $l \rightarrow \infty, E\left(f \mid \mathscr{F}_{n}\right) \in S_{F_{n}}^{1}\left(\mathscr{F}_{n}\right)$, and applying [15, Lemma 1.3], $S_{E\left(F \mid \mathscr{F}_{n}\right)}^{1}\left(\mathscr{F}_{n}\right) \subset$ $S_{F_{n}}^{1}\left(\mathscr{F}_{n}\right)$, which implies $E\left(F \mid \mathscr{F}_{n}\right) \subset F_{n}$ a.s. Q.E.D.

For martingales, in addition to the convergence results in Theorems 2.1, 3.1, and 4.1 in the next section, we have the following Hausdorff convergence result.

Theorem 3.3. Assume that $\left(F_{n}, \mathscr{F}_{n}, n \geq 1\right)$ is an $L^{1}$-bounded martingale, assumption (A) holds, and $F$ constructed in (2.6) is in $\mathbf{L}_{c}^{1}$. If (i), (ii), or (iii) holds: (i) $\left(\left|F_{n}\right|\right)$ is uniformly integrable; (ii) $X^{*}$ is strongly separable; (iii) $\left(F_{n}\right) \subset \mathbf{L}_{c}^{1}$ a.s., then

$$
F_{n} \stackrel{\mathrm{h}}{\rightarrow} F \text { a.s. }
$$

Proof. (3.1) follows from the proof of (d2) in Theorem 2.1, noticing that $\left(s\left(x^{*}, F_{n}\right)-s\left(x^{*}, E\left(F \mid \mathscr{F}_{n}\right)\right), \mathscr{F}_{n}, n \geq 1\right)$ now is a martingale. Q.E.D.

Remark 3.1. Theorem 3.1 was obtained by Hiai and Umegaki (1977) under an additional assumption that $X$ is reflexive or $F(\omega)$ is compact for a.s. $\omega$. They also constructed an example [14, Example 6.6], showing that the condition $F \in \mathbf{L}_{c}^{1}$ cannot be weakened by $F \in \mathscr{L}_{c}^{1}$ even if $X$ is reflexive.

Remark 3.2. (1) (i) $\Rightarrow$ (iii) in Theorem 3.2 was obtained by Hiai and Umegaki (1977) (under an additional condition that $X^{*}$ is strongly separable) and $\mathrm{Pa}$ pageorgiou (1989). (2) When $X$ is the separable dual space of a Banach space, Neveu (1972) proved (i) $\Leftrightarrow$ (iii) $\Leftrightarrow$ (vi) in Theorem 3.2.

Remark 3.3. When $X$ is the separable dual of a Banach space, Neveu (1972) proved (2.10) for $L^{1}$-bounded martingales and proved (3.1) when $F \in \mathbf{L}_{c}^{1}$; Daures (1973) proved (3.1) under conditions: $F_{n}$ is a.s. compact, $n \geq 1$, and $X$ is reflexive; and under the condition $E \sup _{n}\left|F_{n}\right|<\infty$, Papageorgiou (1989) proved $F_{n} \stackrel{\text { K-M }}{\rightarrow} H$ a.s. for some $H \in \mathscr{L}_{c}^{1}$.

\section{ON MULTIVALUED SUPERMARTINGALES}

In Van Cutsem (1972), the author proved convergence theorems for multivalued supermartingales when $X$ is a finite dimensional space. When $X$ is 
a general Banach space, Papageorgiou (1987) proved that if a supermartingale $\left(F_{n}, \mathscr{F}_{n}, n \geq 1\right)$ is contained in $G \in \mathscr{L}_{w k c} \cap \mathscr{L}_{c}^{1}: \bigcup_{n} F_{n} \subset G$ a.s., and if $X^{*}$ is strongly separable, then $F_{n} \stackrel{\mathrm{w}}{\rightarrow} F$ a.s. for some $F \in \mathscr{L}_{c}^{1}$. Recently, Hess (1991) developed a truncation argument in Van Cutsem (1972), and proved a Kuratowski-Mosco convergence theorem for supermartingales with unbounded values when $\bigcup_{n} F_{n} \subset G$ for some $G \in \mathscr{L}_{l w k c}$, where

$$
\mathscr{L}_{l w k c}=\left\{G \in \mathscr{L}_{c}: G \cap B(0, r) \in \mathscr{L}_{w k c}, r>0\right\},
$$

and $B(0, r)$ is the closed ball of radius $r$, centered at 0 .

Definition 4.1. Given $\left(F_{n}\right) \subset \mathscr{L}_{c}{ }^{1}$, we say that (i) assumption $\left(\mathrm{A}^{\prime}\right)$ holds, if $X^{*}$ is strongly separable and there is a subsequence $\left\{n_{k}\right\}$ such that $F_{n_{k}} \in \mathscr{L}_{l w k c}$ and either (a) or (b) in the following holds: (a) $X$ has the Radon-Nikodým property, (b) $\liminf h_{n} h^{+}\left(F_{n}, G\right)=0$ a.s. for some $G \in \mathscr{L}_{l w k c}$; (ii) assumption (B') holds, if $F_{n} \in \mathscr{L}_{l w k c}$ and $h\left(F_{n}, G \cap F_{n}\right) \rightarrow 0$ a.s. for some $G \in \mathscr{L}_{l w k c}$; (iii) assumption $\left(\mathrm{C}^{\prime}\right)$ holds, if there is a subsequence $\left\{n_{k}\right\}$ such that $F_{n_{k}} \in \mathscr{L}_{l w k c}$ and either (a) or the following (c) holds: (c) $\liminf _{k} h^{+}\left(F_{n_{k}}, G\right)=0$ a.s. for some $G \in \mathscr{L}_{l w k c}$.

In this section we prove the following convergence and closedness results for multivalued supermartingales.

Theorem 4.1. Suppose that $\left(F_{n}, \mathscr{F}_{n}, n \geq 1\right)$ is a supermartingale such that $\sup _{n} E d\left(0, F_{n}\right)<\infty$ and assumption $\left(\mathrm{A}^{\prime}\right)$ or $\left(\mathrm{B}^{\prime}\right)$ holds. Then there exists $F \in \mathscr{L}_{c}^{d 1}$ such that

$$
F_{n} \stackrel{\mathrm{K}-\mathrm{M}}{\rightarrow} F \quad \text { a.s. }
$$

(ii) if $\liminf _{n}\left|F_{n}\right|<\infty$,

$$
\lim _{n} s\left(x^{*}, F_{n}\right)=s\left(x^{*}, F\right) \quad \text { a.s. }, \quad x^{*} \in X^{*},
$$

and if $\lim \sup _{n}\left|F_{n}\right|<\infty$ a.s., then

$$
F_{n} \stackrel{\mathrm{W}}{\rightarrow} F
$$

(iii) if for some $G \in \mathscr{L}_{l w k c}, \liminf _{n} h^{+}\left(F_{n}, G\right)=0$ a.s., then for a.s. $\omega \in$ $\Omega, F_{n}(\omega)$ is Wijsman convergent to $F(\omega)$.

Theorem 4.2. Let $\left(F_{n}, \mathscr{F}_{n}, n \geq 1\right)$ be a supermartingale. If assumption $\left(\mathrm{C}^{\prime}\right)$ holds, then there exists $F \in \mathscr{L}_{c}^{d 1}$ such that $E\left(F \mid \mathscr{F}_{n}\right) \subset F_{n}$ a.s. if and only if $\left(d\left(0, F_{n}\right), n \geq 1\right)$ is uniformly integrable.

Remark 4.1. Under the assumption $\bigcup_{n} F_{n} \subset G$ for some $G \in \mathscr{L}_{l w k c}$, Hess (1991) proved that if $\sup _{n} E d\left(0, F_{n}\right)<\infty$, then (4.1) holds; and if $\left(d\left(0, F_{n}\right)\right)$ is uniformly integrable, then supermartingale $\left(F_{n}, \mathscr{F}_{n}, n \geq 1\right)$ is closed. Under the assumption $\bigcup_{n} F_{n} \subset G$ for some $G \in \mathscr{L}_{w k c}$, Hess (1991) also proved (4.3) and the a.s. Wijsman convergence of $F_{n}$ to $F$.

To prove Theorems 4.1 and 4.2 we need the following lemmas.

Lemma 4.1. Suppose that $\left(A_{n}, n \geq 1\right) \subset P_{c}(X), A_{1} \supset A_{2} \supset \cdots$, and $h\left(A_{n}, G \cap A_{n}\right) \rightarrow 0$ for some $G \in P_{w k c}(X)$. Then $A \equiv \bigcap_{n} A_{n} \in P_{w k c}(X)$ and

$$
\lim _{n} s\left(x^{*}, A_{n}\right)=s\left(x^{*}, A\right), \quad x^{*} \in X^{*} .
$$


Proof. It is easy to show that $A \in P_{w k c}(X)$, and $\lim _{n} s\left(x^{*}, A_{n}\right) \geq s\left(x^{*}, A\right)$, $x^{*} \in X^{*}$. Now for any fixed $x^{*} \in X^{*}$, choose $y_{n} \in A_{n} \cap G, s\left(x^{*}, y_{n}\right)>$ $s\left(x^{*}, A_{n}\right)-1 / n-\left\|x^{*}\right\| h\left(A_{n}, A_{n} \cap G\right)$. Then there is a subsequence $\left(y_{n_{k}}, k \geq 1\right)$ such that $y_{n_{k}} \stackrel{\text { w }}{\rightarrow} y$. It is easy to see that $y \in A$ and

$$
\lim _{n} s\left(x^{*}, A_{n}\right)=\lim _{k}\left\langle x^{*}, y_{n_{k}}\right\rangle=\left\langle x^{*}, y\right\rangle \leq s\left(x^{*}, A\right),
$$

and (4.4) holds. Q.E.D.

Lemma 4.2. Suppose that $\left(F_{n}, n \geq 1\right) \subset \mathscr{L}_{c}^{d 1}, F_{1} \supset F_{2} \supset \cdots$ a.s., and $h\left(F_{n}, G \cap F_{n}\right) \rightarrow 0$ a.s. for some $G \in \mathscr{L}_{w k c}$. Then $F \equiv \bigcap_{n} F_{n} \in \mathscr{L}_{w k c}$ and for any sub- $\sigma$-algebra $\mathscr{F} \subset \mathscr{A}$,

$$
s\left(x^{*}, E(F \mid \mathscr{F})\right)=s\left(x^{*}, \bigcap_{n} E\left(F_{n} \mid \mathscr{F}\right)\right) \quad \text { a.s. }, \quad x^{*} \in X^{*} .
$$

And if $\bigcap_{n} E\left(F_{n} \mid \mathscr{F}\right) \in \mathscr{L}_{w k c}$ or $X^{*}$ is strongly separable, then

$$
E(F \mid \mathscr{F})=\bigcap_{n} E\left(F_{n} \mid \mathscr{F}\right) \text { a.s. }
$$

Proof. By Lemma 4.1, $F \in \mathscr{L}_{w k c}$, and for any $x^{*} \in X^{*}$, by Lemma 2.1 and the monotone convergence theorem,

$$
\begin{aligned}
s\left(x^{*}, E(F \mid \mathscr{F})\right) & =E\left(s\left(x^{*}, F\right) \mid \mathscr{F}\right)=E\left(\lim _{n} s\left(x^{*}, F_{n}\right) \mid \mathscr{F}\right) \\
& =\lim _{n} E\left(s\left(x^{*}, F_{n}\right) \mid \mathscr{F}\right)=\lim _{n} s\left(x^{*}, E\left(F_{n} \mid \mathscr{F}\right)\right) \\
& \geq s\left(x^{*}, \bigcap_{n} E\left(F_{n} \mid \mathscr{F}\right)\right) \geq s\left(x^{*}, E(F \mid \mathscr{F})\right) \quad \text { a.s., }
\end{aligned}
$$

(4.5) holds. If $X^{*}$ is strongly separable, then (4.6) follows from (4.5). If $\bigcap_{n} E\left(F_{n} \mid \mathscr{F}\right) \in \mathscr{L}_{w k c}$ a.s., then $\bigcap_{n} E\left(F_{n} \mid \mathscr{F}\right) \supset E(F \mid \mathscr{F}) \in \mathscr{L}_{w k c}$ a.s., and by the continuity in the Mackey topology, (4.5) implies (4.6). Q.E.D.

Definition 4.2. Given $\left(F_{n}\right) \subset \mathscr{L}_{c}^{d 1}$, we say that (i) assumption $\left(\mathrm{A}_{1}^{\prime}\right)$ holds, if $X^{*}$ is strongly separable and if there is a subsequence $\left\{n_{k}\right\}$ such that $F_{n_{k}} \in$ $\mathscr{L}_{w k c}, k \geq 1$; (ii) assumption (B $\left.\mathrm{B}_{1}^{\prime}\right)$ holds, if $F_{n} \in \mathscr{L}_{w k c}, n \geq 1$.

Given a supermartingale $\left(F_{n}, \mathscr{F}_{n}, n \geq 1\right)$, we define $G_{n}=\bigcap_{m \geq n} E\left(F_{m} \mid \mathscr{F}_{n}\right)$.

Lemma 4.3. Let $\left(F_{n}, \mathscr{F}_{n}, n \geq 1\right)$ be a supermartingale satisfying assumption $\left(\mathrm{A}_{1}^{\prime}\right)$ or $\left(\mathrm{B}_{1}^{\prime}\right)$. Then $\left(G_{n}, \mathscr{F}_{n}, n \geq 1\right)$ is a martingale.

Proof. By the definition of supermartingales, we have

$$
F_{n} \supset E\left(F_{n+1} \mid \mathscr{F}_{n}\right) \supset E\left(F_{n+2} \mid \mathscr{F}_{n}\right) \supset \cdots \text { a.s., } \quad n \geq 1 .
$$

If assumption $\left(\mathrm{B}_{1}^{\prime}\right)$ holds, then, by Lemma $4.2, G_{n} \in \mathscr{L}_{w k c}$ and

$$
\begin{aligned}
E\left(G_{n+1} \mid \mathscr{F}_{n}\right) & =E\left(\bigcap_{m \geq n+1} E\left(F_{m} \mid \mathscr{F}_{n+1}\right) \mid \mathscr{F}_{n}\right) \\
& =\bigcap_{m \geq n} E\left(F_{m} \mid \mathscr{F}_{n}\right)=G_{n} \quad \text { a.s. }
\end{aligned}
$$


In the following we assume that $X^{*}$ is strongly separable and there exists a subsequence $\left(n_{k}\right)$ such that $F_{n_{k}} \in \mathscr{L}_{w k c}, k \geq 1$. Then, for any $k \geq 1$, $\bigcap_{m \geq n_{k}} E\left(F_{m} \mid \mathscr{F}_{n_{k}}\right) \in \mathscr{L}_{w k c}$. For any $n \geq 1$, choose $n_{k-1} \geq n$, then, by Lemma 4.2 ,

$$
\begin{aligned}
G_{n} & =\bigcap_{m \geq n} E\left(F_{m} \mid \mathscr{F}_{n}\right)=\bigcap_{m \geq n_{k-1}} E\left(E\left(F_{m} \mid \mathscr{F}_{n_{k-1}}\right) \mid \mathscr{F}_{n}\right) \\
& =E\left(\bigcap_{m \geq n_{k-1}} E\left(F_{m} \mid \mathscr{F}_{n_{k-1}}\right) \mid \mathscr{F}_{n}\right) \in \mathscr{L}_{c}^{d 1},
\end{aligned}
$$

and

$$
\begin{aligned}
E\left(G_{n+1} \mid \mathscr{F}_{n}\right) & =E\left(\bigcap_{m \geq n+1} E\left(F_{m} \mid \mathscr{F}_{n+1}\right) \mid \mathscr{F}_{n}\right) \\
& =E\left(\bigcap_{m \geq n_{k}} E\left(E\left(F_{m} \mid \mathscr{F}_{n_{k}}\right) \mid \mathscr{F}_{n+1}\right) \mid \mathscr{F}_{n}\right) \\
& =E\left(E\left(\bigcap_{m \geq n_{k}} E\left(F_{m} \mid \mathscr{F}_{n_{k}}\right) \mid \mathscr{F}_{n+1}\right) \mid \mathscr{F}_{n}\right) \\
& =E\left(\bigcap_{m \geq n_{k}} E\left(F_{m} \mid \mathscr{F}_{n_{k}}\right) \mid \mathscr{F}_{n}\right)=\bigcap_{m \geq n_{k}} E\left(E\left(F_{m} \mid \mathscr{F}_{n_{k}}\right) \mid \mathscr{F}_{n}\right) \\
& =\bigcap_{m \geq n_{k}} E\left(F_{m} \mid \mathscr{F}_{n}\right)=G_{n} \text { a.s. }
\end{aligned}
$$

$\left(G_{n}, \mathscr{F}_{n}, n \geq 1\right)$ is a martingale. Q.E.D.

For a supermartingale $\left(F_{n}, \mathscr{F}_{n}, n \geq 1\right)$, by Lemma $2.1,\left(d\left(x, F_{n}\right), \mathscr{F}_{n}, n \geq\right.$ $1)$ is a real-valued submartingale. Let $v_{n}=\sup _{m \geq n} E\left(d\left(0, F_{m}\right) \mid \mathscr{F}_{n}\right)$. It is well known that $\left(v_{n}, n \geq 1\right)$ is the martingale part in the Krickeberg decomposition of submartingale $\left(d\left(0, F_{n}\right), \mathscr{F}_{n}, n \geq 1\right), \lim _{n} E v_{n}=\lim _{n} E d\left(0, F_{n}\right)$, and $\left(v_{n}, n \geq 1\right)$ is uniformly integrable if and only if $\left(d\left(0, F_{n}\right), n \geq 1\right)$ is uniformly integrable (cf. [6 and 22]).

In the following we assume that $\left(F_{n}, \mathscr{F}_{n}, n \geq 1\right)$ is a supermartingale satisfying $\sup _{n} E d\left(0, F_{n}\right)<\infty$, and use the following Hess' (1991) truncation:

$$
F_{n}^{k}=F_{n} \cap B\left(0, v_{n}+k\right), \quad k \geq 1,
$$

(since $v_{n} \geq d\left(0, F_{n}\right), F_{n}^{k}$ is not empty).

Lemma 4.4 (Hess (1991)). $\left(F_{n}^{k}, \mathscr{F}_{n}, n \geq 1\right)$ is a supermartingale such that (i) $\sup _{n} E\left|F_{n}^{k}\right| \leq \sup _{n} E v_{n}+k<\infty$; (ii) $\sup _{n}\left|F_{n}^{k}\right| \leq \sup _{n} v_{n}+k<\infty$ a.s.; (iii) if $F_{n}^{k} \stackrel{\mathrm{K}-\mathrm{M}}{\rightarrow} F^{k}, k \geq 1$, then $F_{n} \stackrel{\mathrm{K}-\mathrm{M}}{\rightarrow} \bigcup_{k} F^{k}$ a.s.

Lemma 4.5. (i) If $d\left(x, F_{n}^{k}\right) \rightarrow d\left(x, F^{k}\right)$ a.s., $k \geq 1$, then

$$
d\left(x, F_{n}\right) \rightarrow d\left(x, \bigcup_{k} F^{k}\right) \text { a.s. }
$$


(ii) If $\liminf _{n}\left|F_{n}\right|<\infty$ a.s., and if $s\left(x^{*}, F_{n}^{k}\right) \rightarrow s\left(x^{*}, F^{k}\right)$ a.s., $k \geq 1$, then $s\left(x^{*}, F_{n}\right) \rightarrow s\left(x^{*}, \bigcup_{k} F^{k}\right)$ a.s.

(iii) If $\lim \sup _{n}\left|F_{n}\right|<\infty$ a.s., and if $F_{n}^{k} \stackrel{\mathrm{W}}{\rightarrow} F^{k}$ a.s., $k \geq 1$, then $F_{n} \stackrel{\mathrm{W}}{\rightarrow} \bigcup_{k} F^{k}$ a.s.

Proof. (i) It is easy to show that

$$
\lim _{n} d\left(x, F_{n}\right) \leq \inf _{k} \lim _{n} d\left(x, F_{n}^{k}\right)=\inf _{k} d\left(x, F^{k}\right)=d\left(x, \bigcup_{k} F^{k}\right)
$$

On the other hand, we can choose $x_{n} \in F_{n} \quad\left(x_{n}\right.$ is a function of $\left.\omega\right)$ such that $d\left(x, F_{n}\right) \geq\left\|x-x_{n}\right\|-1 / n$. Then $\left(\left\|x-x_{n}\right\|, n \geq 1\right)$ is bounded and

$$
\lim _{n} d\left(x, F_{n}\right)=\lim _{n}\left\|x-x_{n}\right\| \geq \inf _{k} \lim _{n} d\left(x, F_{n}^{k}\right)=d\left(x, \bigcup_{k} F^{k}\right) \text { a.s. }
$$

(ii) Since $E s\left(x^{*}, F_{n}\right)^{-} \leq E s\left(x^{*}, F_{n}^{k}\right)^{-} \leq\left\|x^{*}\right\| E\left(v_{n}+k\right)$, by Lemma 2.1, $\left(s\left(x^{*}, F_{n}\right), n \geq 1\right)$ and $\left(s\left(x^{*}, F_{n}^{k}\right), n \geq 1\right)$ are supermartingales and converge almost surely. If $\liminf \operatorname{in}_{n}\left|F_{n}\right|<\infty$ a.s., then for a.s. $\omega \in \Omega$, there exists $k=k(\omega) \geq 1$ such that for infinitely many $n \geq 1 \quad F_{n}(\omega)=F_{n}^{k}(\omega)$, and

$$
\begin{aligned}
\lim _{n} s\left(x^{*}, F_{n}\right) & =\sup _{k} \lim _{n} s\left(x^{*}, F_{n}^{k}\right)=\sup _{k} s\left(x^{*}, F^{k}\right) \\
& =s\left(x^{*}, \bigcup_{k} F^{k}\right) \text { a.s., } \quad x^{*} \in X^{*},
\end{aligned}
$$

(ii) holds. (iii) If $\limsup _{n}\left|F_{n}\right|<\infty$ a.s. and if $F_{n}^{k} \stackrel{\mathrm{W}}{\rightarrow} F^{k}$, then the null set in the proof of (ii) can be independent of $x^{*}$, and (iii) holds. Q.E.D.

Proof of Theorem 4.1. By Lemmas 4.4 and 4.5, we may assume that $\sup _{n} E\left|F_{n}\right|$ $<\infty, \sup _{n}\left|F_{n}\right|<\infty$ a.s. and the following (1) or (2) is satisfied: (1) $X^{*}$ is strongly separable and assumption (A) holds; (2) assumption (B) holds. We may also assume that for some subsequence $\left(n_{k}\right), F_{n_{k}} \in \mathscr{L}_{w k c}, k \geq 1$, and in proof of (iii) $\liminf _{n} h^{+}\left(F_{n}, G\right)=0$ a.s. for some $G \in \mathscr{L}_{w k c}$. Let $G_{n}=$ $\bigcap_{m \geq n} E\left(F_{m} \mid \mathscr{F}_{n}\right)$. Then $\left(G_{n}, \mathscr{F}_{n}, n \geq 1\right)$ is an $L^{1}$-bounded martingale satisfying (1) or (2). Applying Theorem 2.1, there is $F \in \mathscr{L}_{c}^{1}$ such that

$$
G_{n} \stackrel{\mathrm{W}}{\rightarrow} F \text { a.s. and } G_{n} \stackrel{\mathrm{K}-\mathrm{M}}{\rightarrow} F \text { a.s. }
$$

For any $n \geq 1$, choose $k \geq 1$ such that $n_{k} \geq n$. Then, by Lemmas 2.1, 4.1, 4.2 and the monotone convergence theorem for conditional expectations,

$$
\begin{aligned}
s\left(x^{*}, G_{n}\right)=s\left(x^{*}, \bigcap_{m \geq n_{k}} E\left(E\left(F_{m} \mid \mathscr{F}_{n_{k}}\right) \mid \mathscr{F}_{n}\right)\right) \\
\quad=s\left(x^{*}, E\left(\bigcap_{m \geq n_{k}} E\left(F_{m} \mid \mathscr{F}_{n_{k}}\right) \mid \mathscr{F}_{n}\right)\right)=E\left(s\left(x^{*}, \bigcap_{m \geq n_{k}} E\left(F_{m} \mid \mathscr{F}_{n_{k}}\right)\right) \mid \mathscr{F}_{n}\right) \\
=E\left(\lim _{m} s\left(x^{*}, E\left(F_{m} \mid \mathscr{F}_{n_{k}}\right)\right) \mid \mathscr{F}_{n}\right)=\lim _{m} E\left(s\left(x^{*}, E\left(F_{m} \mid \mathscr{F}_{n_{k}}\right)\right) \mid \mathscr{F}_{n}\right) \\
=\lim _{m} s\left(x^{*}, E\left(E\left(F_{m} \mid \mathscr{F}_{n_{k}}\right) \mid \mathscr{F}_{n}\right)\right)=\lim _{m} s\left(x^{*}, E\left(F_{m} \mid \mathscr{F}_{n}\right)\right) \\
=\lim _{m} E\left(s\left(x^{*}, F_{m}\right) \mid \mathscr{F}_{n}\right) \text { a.s., }
\end{aligned}
$$


and $\left(s\left(x^{*}, F_{n}\right), \mathscr{F}_{n}, n \geq 1\right)$ is a real-valued supermartingale satisfying

$$
\sup _{n} E\left|s\left(x^{*}, F_{n}\right)\right| \leq\left\|x^{*}\right\| \sup _{n} E\left|F_{n}\right|<\infty .
$$

Hence $\left(s\left(x^{*}, F_{n}\right), \mathscr{F}_{n}, n \geq 1\right)$ is a amart, of course, subpramart (cf. Millet and Sucheston (1980)), and

$$
s\left(x^{*}, G_{n}\right)=\lim _{m} E\left(s\left(x^{*}, F_{m}\right) \mid \mathscr{F}_{n}\right)=\text { ess } \inf _{t \in T(n)} E\left(s\left(x^{*}, F_{t}\right) \mid \mathscr{F}_{n}\right) \quad \text { a.s. }
$$

Applying (4.7) and Lemma 2.2, we have

$$
s\left(x^{*}, F\right)=\lim _{n} s\left(x^{*}, G_{n}\right)=\lim _{n} s\left(x^{*}, F_{n}\right) \quad \text { a.s. }, \quad x^{*} \in X^{*},
$$

(4.2) holds. As the proof of (b) in Theorem 2.1, we get (4.1) and (4.3) from (4.2), noticing that

$$
s-\liminf _{n} F_{n} \supset s-\liminf _{n} G_{n}=F \quad \text { a.s. }
$$

Proof of (iii). Since there is $G \in \mathscr{L}_{w k c}$ such that $\liminf _{n} h^{+}\left(G_{n}, G\right) \leq$ $\lim \inf _{n} h^{+}\left(F_{n}, G\right)=0$ a.s., $G \supset F \in \mathscr{L}_{w k c}$ a.s. (see the proof of Lemma 2.4). By Lemma 2.4 and its proof, $\lim _{n} d\left(x, F_{n}\right) \leq \lim _{n} d\left(x, G_{n}\right)=d(x, F)$ a.s. and

$$
\begin{aligned}
\lim _{n} d\left(x, F_{n}\right) & \geq \lim \sup _{n} \sup _{x^{*} \in B^{*}}\left(\left\langle x^{*}, x\right\rangle-s\left(x^{*}, F_{n}\right)\right) \\
& \geq \sup _{x^{*} \in M^{*}}\left(\left\langle x^{*}, x\right\rangle-s\left(x^{*}, F\right)\right)=d(x, F) \text { a.s. , }
\end{aligned}
$$

(iii) holds. Q.E.D.

Proof of Theorem 4.2. Assume that for some subsequence $\left(n_{k}\right), F_{n_{k}} \in \mathscr{L}_{l w k c}$, $k \geq 1$. If $\left(d\left(0, F_{n}\right)\right)$ is uniformly integrable, then $\left(v_{n}\right)$, and hence $\left(\left|F_{n}^{j}\right|\right)$, is uniformly integrable, $j \geq 1$. Let $G_{n_{k}}^{j}=\bigcap_{m \geq n_{k}} E\left(F_{m}^{j} \mid \mathscr{F}_{n_{k}}\right)$. Then, by Lemmas 4.4 and $4.3,\left(G_{n_{k}}^{j}, \mathscr{F}_{n_{k}}, k \geq 1\right)$ is a uniformly integrable martingale satisfying assumption (A), and by Theorem 3.2 , there is $F^{j} \in \mathscr{L}_{c}^{1}$ such that $G_{n_{k}}^{j}=$ $E\left(F^{j} \mid \mathscr{F}_{n_{k}}\right)$ a.s. For each $n \geq 1$ choose $k \geq 1$ such that $n_{k} \geq n$, then

$$
\begin{aligned}
F_{n}^{j} & \supset E\left(F_{n_{k}}^{j} \mid \mathscr{F}_{n}\right) \supset E\left(G_{n_{k}}^{j} \mid \mathscr{F}_{n}\right) \\
& =E\left(E\left(F^{j} \mid \mathscr{F}_{n_{k}}\right) \mid \mathscr{F}_{n}\right)=E\left(F^{j} \mid \mathscr{F}_{n}\right) \text { a.s. }
\end{aligned}
$$

Let $F=\bigcup_{j} F^{j}$. Then $F \in \mathscr{L}_{c}^{d 1}$, and, by [14, Theorem 2.1],

$$
F_{n}=\bigcup_{j} F_{n}^{j} \supset \mathrm{cl}\left(\bigcup_{j} E\left(F^{j} \mid \mathscr{F}_{n}\right)\right)=E\left(\bigcup_{j} F^{j} \mid \mathscr{F}_{n}\right)=E\left(F \mid \mathscr{F}_{n}\right) \text { a.s. }
$$

On the other hand, if (4.9) holds for some $F \in \mathscr{L}_{c}^{d 1}$, then, by Lemma 2.1,

$$
d\left(0, F_{n}\right) \leq d\left(0, E\left(F \mid \mathscr{F}_{n}\right)\right) \leq E\left(d(0, F) \mid \mathscr{F}_{n}\right),
$$

$\left(d\left(0, F_{n}\right)\right)$ is uniformly integrable. Q.E.D.

\section{ACKNOWLEDGMENT}

The authors are grateful to the referee for most helpful comments. 


\section{REFERENCES}

1. R. Alo, A. de Korvin, and C. Roberts, The optional sampling theorem for convex set valued martingales, J. Math. 310 (1979), 1-6.

2. J. Aubin and H. Frankowska, Set-valued analysis, Birkhäuser, Boston, Mass., 1990.

3. A. Bellow, Uniform amarts: $A$ class of asymptotic martingales for which strong almost sure convergence obtains, Z. Wahr. Verw. Gebiete 41 (1978), 177-191.

4. C. Castaing and M. Valadier, Convex analysis and measurable multifunctions, Lecture Notes in Math., vol. 580, Springer-Verlag, Berlin and New York, 1977.

5. S. Chatterji, Vector values martingales and their applications, Lecture Notes in Math., vol. 526, Springer-Verlag, Berlin and New York, 1976, pp. 33-51.

6. Y. S. Chow, H. Robbins, and D. Siegmund, Great expectation: The theory of optimal stopping, Houghton Mifflin, New York, 1971.

7. F. Clarke, Optimization and nonsmooth analysis, Wiley, New York, 1984.

8. J. P. Daures, Version multivoque du théorème de Doob, Ann. Inst. Henri Poincaré B 9 (1973), 167-176.

9. L. Egghe, Stopping time techniques for analysts and probabilists, Cambridge Univ. Press, London and New York, 1984.

10. S. Francaviglia, A. Lechicki, and S. Levi, Quasi-uniformization of hyperspaces and convergence of nets of semicontinuous multifunctions, J. Math. Anal. Appl. 112 (1985), 347-370.

11. N. Ghoussoub and L. Sucheston, $A$ refinement of the Riesz decomposition for amarts and semiamarts, J. Multivariate Anal. 8 (1978), 146-150.

12. C. Hess, On multivalued martingales whose values may be unbounded: martingale selectors and Mosco convergence, J. Multivariate Anal. 39 (1991), 175-201.

13. F. Hiai, Strong laws of large numbers for multivalued random variables, Lecture Notes in Math., vol. 1091, Springer-Verlag, Berlin and New York, 1984, pp. 160-172.

14. Convergence of conditional expectations and strong laws of large numbers for multivalued random variables, Trans. Amer. Math. Soc. 291 (1985), 613-627.

15. F. Hiai and H. Umegaki, Integrals, conditional expectations, and martingales of multivalued functions, J. Multivariate Anal. 7 (1977), 149-182.

16. W. Hildenbrand, Core and equilibria of a large economy, Princeton Univ. Press, Princeton, N.J., 1974.

17. C. Himmelberg and F. Van Vleck, Multifunctions on abstract measurable space and application to stochastic decision theory, Ann. Mat. Pura. Appl. 101 (1974), 229-236.

18. A. de Korvin and R. Kleyle, A convergence theorem for convex set valued supermartingales, Stochastic Anal. Appl. 3 (1985), 433-445.

19. A. Lechicki and S. Levi, Wijsman convergence in the hyperspace of a metric space, Boll. Un. Mat. Ital. (7) 1-B (1987), 439-451.

20. A. Millet and L. Sucheston, Convergence of classes of amart indexed by directed sets, Canad. J. Math. 32 (1980), 86-125.

21. N. Neveu, Convergence presque sur de martingales multivogues, Ann. Inst. Henri Poincaré B 8 (1972), 1-7.

22. __ Discrete parameter martingales, North-Holland, Amsterdam, 1975.

23. N. S. Papageorgiou, On the theory of Banach space valued multifunctions. Part 1: Integration and conditional expectation, J. Multivariate Anal. 17 (1985a), 185-206.

24. __ On the theory of Banach space valued multifunctions. Part 2: Set valued martingales and set valued measures, J. Multivariate Anal. 17 (1985b), 207-227.

25. $\ldots$ On the efficiency and optimality of allocations. II, SIAM. J. Control Optim. 24 (1986), 452-479.

26. _ $\ldots$ A convergence theorem for set valued supermartingales with values in parable Banach space, Stochastic Anal. Appl. 5 (1987), 510-520. 
27. Convergence and representation theorems for set valued random processes, Stochastics Anal. Appl. 7 (1989), 187-210.

28. H. Robbins, On the measure of a random set, Ann. Math. Statist. 15 (1944), 70-74.

29. $\ldots$, On the measure of a random set (II), Ann. Math. Statist. 16 (1945), 342-347.

30. G. Salinetti and R. Wets, On convergence of sequences of convex sets in finite dimensions, SIAM Rev. 21 (1979), 18-23.

31. B. Van Cutsem, Martingales de multiapplications à valeurs convexes compactes, $\mathbf{C}$. R. Acad. Sci. Paris 269 (1969), 429-432.

32. _ Martingales de convexes fermés aléatoires en dimension finite, Ann. Inst. Henri Poincaré B 8 (1969), 365-385.

33. R. A. Wijsman, Convergence of sequences of convex sets, cones and functions. II, Trans. Amer. Math. Soc. 123 (1966), 32-45.

34. M. C. Yovits, C. R. Foulk, and L. Rose, Information flow and analysis: Theory, simulation and experiment. Part I: Basic theoretical and conceptual development, Amer. Soc. Inform. Sci. 32 (1981), 187-202.

Department of Statistics, East China Normal University, Shanghai 200062, China

Department of Statistics, Columbia University, New York, New York 10027 